\title{
Buckling strength topology optimization of 2D periodic materials based on linearized bifurcation analysis
}

Thomsen, Christian Rye; Wang, Fengwen; Sigmund, Ole

\section{Published in:}

Computer Methods in Applied Mechanics and Engineering

Link to article, DOI:

10.1016/j.cma.2018.04.031

Publication date:

2018

Document Version

Peer reviewed version

Link back to DTU Orbit

Citation (APA):

Thomsen, C. R., Wang, F., \& Sigmund, O. (2018). Buckling strength topology optimization of 2D periodic materials based on linearized bifurcation analysis. Computer Methods in Applied Mechanics and Engineering, 339, 115-136. https://doi.org/10.1016/j.cma.2018.04.031

\section{General rights}

Copyright and moral rights for the publications made accessible in the public portal are retained by the authors and/or other copyright owners and it is a condition of accessing publications that users recognise and abide by the legal requirements associated with these rights.

- Users may download and print one copy of any publication from the public portal for the purpose of private study or research.

- You may not further distribute the material or use it for any profit-making activity or commercial gain

- You may freely distribute the URL identifying the publication in the public portal 


\section{Accepted Manuscript}

Buckling strength topology optimization of $2 \mathrm{D}$ periodic materials based on linearized bifurcation analysis

Christian Rye Thomsen, Fengwen Wang, Ole Sigmund

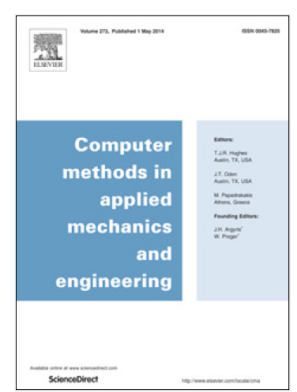

PII:

S0045-7825(18)30210-X

DOI: $\quad$ https://doi.org/10.1016/j.cma.2018.04.031

Reference: CMA 11882

To appear in: Comput. Methods Appl. Mech. Engrg.

Received date : 8 December 2017

Revised date: 16 April 2018

Accepted date : 18 April 2018

Please cite this article as: C.R. Thomsen, F. Wang, O. Sigmund, Buckling strength topology optimization of 2D periodic materials based on linearized bifurcation analysis, Comput. Methods Appl. Mech. Engrg. (2018), https://doi.org/10.1016/j.cma.2018.04.031

This is a PDF file of an unedited manuscript that has been accepted for publication. As a service to our customers we are providing this early version of the manuscript. The manuscript will undergo copyediting, typesetting, and review of the resulting proof before it is published in its final form. Please note that during the production process errors may be discovered which could affect the content, and all legal disclaimers that apply to the journal pertain. 


\title{
Buckling strength topology optimization of 2D periodic materials based on linearized bifurcation analysis
}

\author{
Christian Rye Thomsen, Fengwen Wang*, Ole Sigmund \\ Department of Mechanical Engineering, Technical University of Denmark, Nils Koppels Allé, Building 404, 2800 Kgs. Lyngby, Denmark
}

\begin{abstract}
Low density cellular materials may offer excellent mechanical properties and find wide applicability in lightweight design and infill structures for additive manufacturing, yet currently existing material structures are still far away from their theoretical limit in terms of compressive strength. To explore the existing potential, this paper presents a topology optimization framework for designing periodic cellular materials with maximized strength under compressive loading. Under this condition, the limiting factor of strength is the failure mechanism of buckling instability in the microstructure. In order to predict microstructural buckling, a simplified model based on homogenization theory, a linearized stability criterion and Floquet-Bloch theory is employed. Subsequently, a gradient-based topology optimization problem is formulated to maximize the buckling strength of the most critical failure mode. The framework is utilized to optimize square, triangular and hexagonal microstructures for three different macroscopic load conditions including biaxial, uniaxial and shear loading, and performance assessments are conducted by computation of associated failure surfaces in macroscopic stress space. In all cases, the optimized designs turn out to be first-order hierarchical type microstructures which offer major improvements of strength compared to the initial zero-order designs, however, the gains come at the cost of reductions in stiffness. Furthermore, it is illustrated how imposing geometric symmetry constraints can be exploited to control the shape of the failure surfaces.
\end{abstract}

Keywords: Topology optimization, Periodic materials, Microstructural buckling instability, Floquet-Bloch theory, Macroscopic stress loading

\section{Introduction}

In recent years, artificially constructed materials have experienced a tremendous growth in popularity due to their large potential and customizability for a widespread range of applications, and due to the increased fabrication accessibility following advancement in additive manufacturing. This has resulted in a multitude of exotic materials which offer desirable properties ranging from low density with high strength, stiffness and recoverability $[1,2,3]$ to excellent shock absorption capabilities [4, 5], wave-guiding [6], and materials which harness localized instability mechanisms to control the material properties $[7,8,9]$. The reader is referred to a recent review paper for additional references on the topic [10].

Of particular interest here is the subject of maximizing material strength in lightweight cellular materials through intelligent design of the microstructure. This constitutes a highly relevant objective, as material design without consideration of local stability is essentially only of theoretical interest, and as the current state of art is still orders of magnitude away from the theoretical limit of achievable strength [11]. For lightweight materials, the strength capacity is limited by the failure mechanism of microstructural buckling instability which occurs when its slender structural members are subjected to compressive loading. As this failure mode is highly dependent on geometry, previous studies have investigated a wide range of microstructure topologies in search for increased stability, including 2D honeycombs [12], lattice structures [13] and thin-walled structures [14]. Furthermore, studies have discovered certain

\footnotetext{
${ }^{*}$ Corresponding author.

Email address: f wan@mek.dtu.dk (Fengwen Wang)
} 
geometric attributes which yield high performance in the design for stability. This includes closed-walled shell structures such as hollow-tubed lattices [1], as well as hierarchical structures where the geometrical details span across multiple length scales [15]. Specifically the hierarchical approach has demonstrated a tremendous potential, e.g. in work by Meza et al. [2] and Zheng et al. [16] which fabricated multi-level hierarchical lattice structures that strongly outperformed their non-hierarchical counterparts. Nevertheless, the limit of strength has yet to be realized, and strong incentives exist to explore the potential for further optimization.

Modelling of buckling instability in the elastic response of materials with periodic microstructures is a complex subject matter which has received much interest in the past decades. Instabilities may develop with different wavelengths depending on the geometry of the microstructure and macroscopic load conditions, spanning from highly localized modes on the microscale to long wavelength modes on the macroscale. In addition, further complications involve scale effects, boundary effects due to the finite cell size, material non-linearities from e.g. plasticity or ratedependency, and geometrical non-linearities. With the desire to reduce model complexity, previous numerical studies have resorted to simplified models which employ homogenization methods for separating scales [17]. For modelling of instabilities developing on the microscale, the Bloch-wave technique has proven to be very effective, as it retains the analysis to the repetitive unit cell of the microstructure while capturing all wavelengths of instability with imposed periodicity constraints $[18,19]$. In extension to this, Triantafyllidis and Schnaidt [20] have proposed a strong methodology for characterizing the material strength, which utilizes the concept of failure surfaces to represent microstructural instability for arbitrary macroscopic stress states. Lately, this method was used by Haghpanah et al. [21] to form analytic expressions for the failure surfaces of simple honeycomb microstructures. Other studies have been focused on investigating the influence of scale effects and microscopic imperfections [22] and advanced homogenization models with non-linear material models [23].

In the field of material design, the method of inverse homogenization originally introduced by Sigmund [24] has found common use. The method utilizes topology optimization [25] to find the optimized distribution of material within the unit cell of the periodic microstructure such that specified macroscopic properties are achieved. The method has been successfully applied to multiple design problems, including materials with extremal thermal properties [26], extremal elastic properties such as negative Poisson's ratio [27, 28, 29] or maximal bulk and shear modulus [30, 31], and for materials with vibration mitigation abilities and phononic band gaps [6]. The latter example is comparable to the buckling instability problem as it also adopts Bloch-wave boundary conditions. Design for strength was initially considered by Bendsøe and Triantafyllidis [32], who formulated a sizing optimization problem to increase the microstructural buckling strength for a rectangular orthogrid. Subsequently, the problem was considered in a topology optimization formulation by Neves et al. [33], but that study only treated localized instability modes with cell-periodic wavelengths. The work was later extended to cover non-local modes with the Bloch-wave technique in [34]. The interested reader is referred to a recent review paper for additional references to the field of architected material design by topology optimization [35].

In design for stability with topology optimization, multiple computational challenges will be faced. The topic was first considered in [36] based on the linear stability eigenvalue problem, addressing the complications associated with localized spurious modes and non-differentiability of eigenvalues with multiplicity in gradient-based approaches [37]. More recently, optimization with linear stability constraints has been applied to lattice structures [38], models including plasticity effects [39] and 3D problems based on the level set method [40]. Other studies have employed more elaborate stability models which account for large deformations through geometrical non-linearities for both solid structures [41] and composites [42]. Furthermore, studies have addressed the influence of geometric imperfections from manufacturing errors and their potentially detrimental effects on stability. Proposed techniques for reducing imperfection sensitivity utilize small structural misalignments in the optimization [43], or more comprehensive stochastic models based on Monte Carlo simulations to cover a wider range of geometric uncertainties [44].

The present paper seeks to explore the existing strength potential, and design novel 2D materials with maximum strength against microstructural buckling by use of topology optimization. Accordingly, this paper is an extension of the works by Neves et al. $[33,34]$ where the complete Bloch-wave analysis is incorporated into the stability model, and where the optimization is conducted with highly increased resolution and a more refined regularization technique. The employed model is based on linear elasticity, linear homogenization theory, a linearized stability condition and Bloch-wave analysis to predict microstructural buckling for prescribed macroscopic load conditions. This choice of model ensures high computational efficiency, but also comes with significant limitations that need to be addressed. The validity of the homogenization approach is based on the assumption that the scale of the microstructure is infinitely 
small compared to the macrostructure and that the microstructure has infinite extend, thus disregarding any scaleand boundary effects in periodic structures of finite size. Furthermore, utilization of a linearized stability criterion imposes a restriction of small strain fields prior to instability, and will disregard any non-linear stiffness altering effects stemming from imperfections in the geometry, base material and load state. It thus only provides a crude estimate of the real strength, but does not require perturbations to trigger instability and reduces the complexity substantially. Despite considerable simplifications, the computational problem still retains an extensive size, and combined with the complications associated with the linear stability optimization problem, it remains a challenging task to be solved.

The paper is structured as follows. In section 2, the model for predicting instability in the material microstructure is presented. This includes the governing equations in continuous form and the finite element discretization for numerical implementation. Section 3 outlines the formulation of the optimization problem including the design parametrization and sensitivity analysis, while Section 4 provides an overview of the computational model. The results are presented in Section 5, showcasing strength optimized designs for the cases of biaxial, uniaxial and shear loading along with a performance assessment and comparison to existing designs. The paper ends with conclusions in Section 6.

\section{Linearized stability model}

The linear elasticity model considers a 2-dimensional macrostructure composed of a cellular material with a perfectly periodic microstructure with representative unit cell $Y$ as shown in Figure 1. Furthermore, it is assumed that a clear separation of length scales exist, i.e. $d / D \ll 1$, such that the homogenization technique and underlying asymptotic expansion is valid. The problem of predicting microstructural buckling instability for a prescribed macroscopic stress state $\bar{\sigma}_{i j}$ can then be decomposed into 2 sub-problems: 1) a homogenization problem, and 2) an instability problem on the microscale. A third problem related to buckling instability on the macroscale exists, but is beyond the scope of the present paper. An in-depth description of the standard homogenization problem can be found in [17] and its extension to microstructural stability in [33]. Here, the focus is on microstructural optimization, and hence we present only the main results necessary for the optimization formulation while referring the readers to aforementioned works for the detailed derivations.

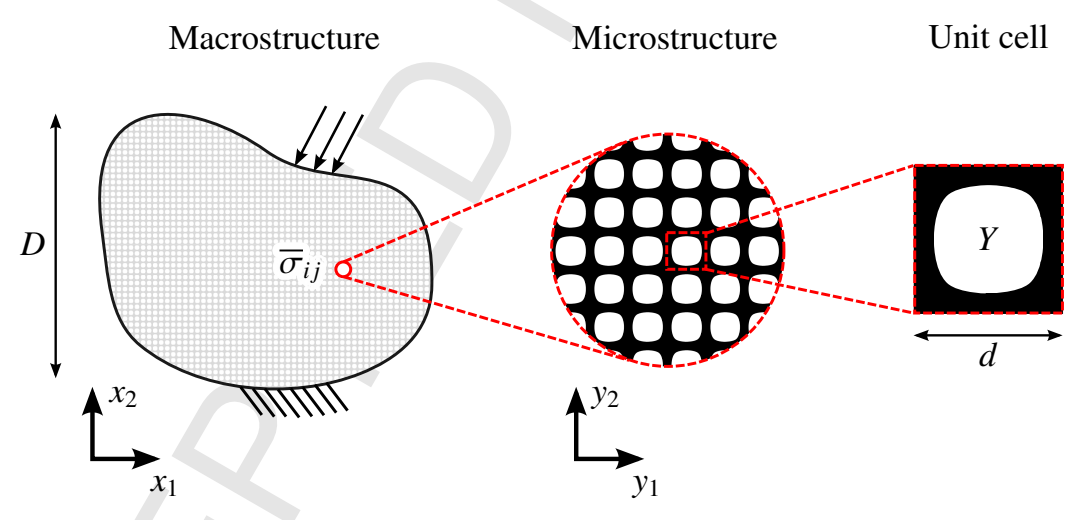

Figure 1: Schematic representation of the length scale separation: the macrostructure in which a macroscopic stress state $\bar{\sigma}_{i j}$ exist due to externally applied loads, and the microstructure which is described by a repetition of unit cell $Y$.

\subsection{Governing equations}

In the homogenization problem, a set of $k, l=1,2$ equilibrium equations on the microscale is first solved to find $Y$-periodic test fields $\chi_{p}^{k l}$ :

$$
\int_{Y} E_{i j p q}(\mathbf{y}) \frac{\partial \chi_{p}^{k l}}{\partial y_{q}} \frac{\partial v_{i}}{\partial y_{j}} d Y=\int_{Y} E_{i j k l}(\mathbf{y}) \frac{\partial v_{i}}{\partial y_{j}} d Y,
$$

where $E_{i j p q}$ is the locally varying constitutive tensor of the base material, and $\mathbf{v}=\left(v_{1}, v_{2}\right)^{T}$ is a kinematically admissible $Y$-periodic virtual displacement field. The displacement fields $\chi_{p}^{k l}$ describe the local variation over the unit cell 
when it is subjected to the $k, l$ prescribed unit strains $\tilde{\varepsilon}_{p q}^{k l}=\delta_{p k} \delta_{q l}$ (which correspond to body loads $\sigma_{i j}^{k l}=E_{i j p q} \tilde{\varepsilon}_{p q}^{k l}=$ $E_{i j k l}$ ), and will be non-zero for inhomogeneous unit cells. Due to symmetry in $E_{i j k l}$ only 3 linearly independent load cases exist, and the equations only need to be solved for cases $k, l=11,22,12$ which correspond to two tensile and one shear test.

Subsequently, the homogenized material properties $\bar{E}_{i j k l}$ can be computed by:

$$
\bar{E}_{i j k l}=\frac{1}{|Y|} \int_{Y}\left(E_{i j k l}(\mathbf{y})-E_{i j p q}(\mathbf{y}) \frac{\partial \chi_{p}^{k l}}{\partial y_{q}}\right) d Y,
$$

where the overline denotes an averaging over the base cell, and $|Y|$ is the volume of the unit cell. The equation can also be expressed more conveniently in an equivalent energy based form:

$$
\bar{E}_{i j k l}=\frac{1}{|Y|} \int_{Y} E_{p q r s}(\mathbf{y})\left(\tilde{\varepsilon}_{r s}^{i j}-\varepsilon_{r s}^{i j}\right)\left(\tilde{\varepsilon}_{p q}^{k l}-\varepsilon_{p q}^{k l}\right) d Y
$$

where $\varepsilon_{p q}^{k l}=\frac{1}{2}\left(\frac{\partial \chi_{p}^{k l}}{\partial y_{q}}+\frac{\partial \chi_{q}^{k l}}{\partial y_{p}}\right)$ is the Cauchy strain tensor of the test fields.

The second problem is concerned with predicting buckling instability on the microscale, and is governed by the following eigenvalue problem:

$$
\int_{Y} E_{i j k l}(\mathbf{y}) \frac{\partial \phi_{i}}{\partial y_{j}} \frac{\partial w_{k}}{\partial y_{l}} d Y-P \int_{Y} \sigma_{i j}(\mathbf{y}) \frac{\partial \phi_{m}}{\partial y_{i}} \frac{\partial w_{m}}{\partial y_{j}} d Y=0,
$$

where $\mathbf{w}=\left(w_{1}, w_{2}\right)^{T}$ is an admissible Floquet-periodic virtual displacement field, $P$ represents the eigenvalues which correspond to the bifurcation loads when multiplied by the macroscopic initial stress $\bar{\sigma}_{i j}$, $\phi_{m}$ denotes associated mode shapes, and $\sigma_{i j}$ is the initial stress in the microstructure prior to bifurcation which is given by:

$$
\sigma_{i j}=\left(E_{i j k l}(\mathbf{y})-E_{i j p q}(\mathbf{y}) \frac{\partial \chi_{p}^{k l}}{\partial y_{q}}\right) \bar{\varepsilon}_{k l} .
$$

where $\bar{\varepsilon}_{k l}$ is the macroscopic strain which is obtained from the macroscopic constitutive equation $\bar{\sigma}_{i j}=\bar{E}_{i j k l} \bar{\varepsilon}_{k l}$.

To model buckling instabilities spanning over several unit cells, the instability equation is supplemented with the Floquet-Bloch condition on the bifurcated modes:

$$
\phi_{m}\left(\mathbf{y}+\mathbf{R}_{j}\right)=\phi_{m}(\mathbf{y}) e^{i \mathbf{k}^{T} \mathbf{R}_{j}}, \quad j=1,2
$$

where $i$ refers to the complex unity $\sqrt{-1}, \mathbf{k}=\left(k_{1}, k_{2}\right)^{T}$ is the wavevector and $\mathbf{R}_{j}$ are primitive lattice vectors which define the geometry of the unit cell, see Figure $2 \mathrm{a}$. Here, both quadratic and rhombic unit cells are considered which have lattice vectors $\mathbf{R}_{1}=(d, 0)^{T}, \mathbf{R}_{2}=(0, d)^{T}$ and $\mathbf{R}_{1}=(d, 0)^{T}, \mathbf{R}_{2}=(d / 2, \sqrt{3} d / 2)^{T}$, respectively. The periodicity constraint ensures that all possible wavelengths of bifurcation are covered when the stability equation is solved for

(a)

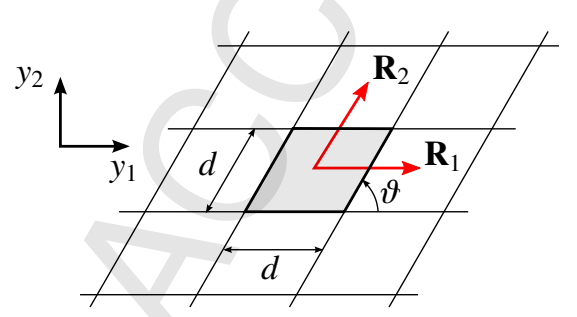

(b)

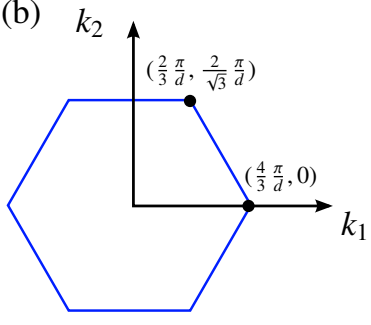

(c) $\quad k_{2}$

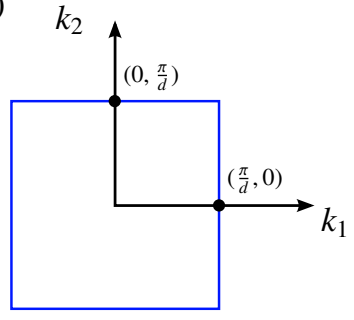

Figure 2: (a) $3 \times 3$ unit cell assembly for a rhombic $\left(\vartheta=60^{\circ}\right)$ and square lattice $\left(\vartheta=90^{\circ}\right)$ with indication of the primitive lattice vectors $\mathbf{R}_{j}$. The associated Brillouin zone is shown in (b) for the rhombic lattice, and in (c) for the square lattice. 
wavevectors spanning the Brillouin zone as shown in Figures 2(b)-(c), and the wavevector with the smallest bifurcation load then defines the wavelength of the microstructural failure mode. However, in many cases the full buckling analysis involving a complete domain investigation can be reduced by exploiting symmetry patterns in the Brillouin zone, which may exist due to symmetries in geometry and macroscopic load state. More specifically, numerical studies have shown (but not proven) that it may be sufficient to investigate only the boundary of the irreducible part of the Brillouin zone and then use band structure diagrams to represent the buckling characteristics, i.e. a similar approach to that used for wave propagation problems [45]. The present work will exploit this reduction method during optimization, and only conduct a complete analysis on the final designs to verify that the assumed symmetries remain valid also for the final design.

\subsection{Finite element formulation}

The governing equations will be solved numerically using the finite element method. The domain of the unit cell is discretized by bilinear Q4 elements in an isoparametric formulation, and element integration is performed using full Gauss point integration. Furthermore, the symmetry properties of $E_{i j k l}$ are exploited to make use of the abbreviation $k l \rightarrow j: 11 \rightarrow 1,22 \rightarrow 2,(12,21) \rightarrow 3$ to represent the equations on more compact form.

\subsubsection{Discretized equations}

The microscale equilibrium equations in (1), which provide the three test fields $\chi^{j}$, have the discretized form:

$$
\mathbf{K} \chi^{j}=\mathbf{f}^{j}, \quad j=1,2,3
$$

where the global stiffness matrix $\mathbf{K}$ and equivalent load vectors $\mathbf{f}^{j}$ are given by:

$$
\mathbf{K}=\sum_{e=1}^{N} \int_{Y_{e}} \mathbf{B}_{e}^{T} \mathbf{E}_{e} \mathbf{B}_{e} d Y, \quad \mathbf{f}^{j}=\sum_{e=1}^{N} \int_{Y_{e}} \mathbf{B}_{e}^{T} \mathbf{E}_{e} \tilde{\boldsymbol{\varepsilon}}^{j} d Y,
$$

where the sum represents a finite element assembly operation over $N$ elements, $\mathbf{B}_{e}$ is the strain-displacement matrix of element $e, \mathbf{E}_{e}$ is the constitutive matrix, and $\tilde{\varepsilon}_{i}^{j}=\delta_{i j}$ denotes the 3 independent unit strain fields. The homogenized constitutive matrix is then calculated by:

$$
\bar{E}_{i j}=\frac{1}{|Y|} \sum_{e=1}^{N} \int_{Y_{e}}\left(\tilde{\boldsymbol{\varepsilon}}^{i}-\mathbf{B}_{e} \mathcal{X}_{e}^{i}\right)^{T} \mathbf{E}_{e}\left(\tilde{\boldsymbol{\varepsilon}}^{j}-\mathbf{B}_{e} \chi_{e}^{j}\right) d Y,
$$

The microstructural instability equation given in (4) has the discretized form:

$$
(\mathbf{K}-P \mathbf{G}) \boldsymbol{\phi}=\mathbf{0},
$$

where $P, \boldsymbol{\phi}$ are the bifurcation loads and mode shapes, and $\mathbf{G}$ is the initial stress stiffness matrix which is given by:

$$
\mathbf{G}=\sum_{e=1}^{N} \int_{Y_{e}} \frac{\partial \mathbf{N}^{T}}{\partial y_{i}}\left(\sigma_{i j}\right)_{e} \frac{\partial \mathbf{N}}{\partial y_{j}} d Y
$$

where $\frac{\partial \mathbf{N}}{\partial y_{i}}$ is the shape function matrix derivative, and summation over indices $i, j=1,2$ is implied. The components of the initial stress tensor $\left(\sigma_{i j}\right)_{e}$ of element $e$ are obtained by:

$$
\sigma_{e}=\mathbf{E}_{e}\left(\mathbf{I}-\mathbf{B}_{e} \boldsymbol{X}_{e}\right) \bar{\varepsilon}
$$

where $\boldsymbol{X}_{e}=\left[\begin{array}{lll}\chi_{e}^{1} & \chi_{e}^{2} & \chi_{e}^{3}\end{array}\right]$ is a $8 \times 3$ matrix containing the element test fields, and $\overline{\boldsymbol{\varepsilon}}=\overline{\mathbf{E}}^{-1} \overline{\boldsymbol{\sigma}}$ is the macroscopic strain field. 


\subsubsection{Boundary conditions}

To facilitate implementation of the periodicity constraints on the microscale equilibrium and instability equations, the boundary node displacements $\mathbf{u}$ (which represent either $\chi$ or $\phi$ ) are numbered as shown in Figure 3 . The periodicity constraints can then be established by use of equation (6):

$$
\begin{gathered}
\mathbf{u}_{r}=e^{i \mathbf{k}^{T} \mathbf{R}_{1}} \mathbf{u}_{l}, \quad \mathbf{u}_{t}=e^{i \mathbf{k}^{T} \mathbf{R}_{2}} \mathbf{u}_{b}, \\
\mathbf{u}_{r b}=e^{i \mathbf{k}^{T} \mathbf{R}_{1}} \mathbf{u}_{l b}, \quad \mathbf{u}_{l t}=e^{i \mathbf{k}^{T} \mathbf{R}_{2}} \mathbf{u}_{l b}, \quad \mathbf{u}_{r t}=e^{i \mathbf{k}^{T}\left(\mathbf{R}_{1}+\mathbf{R}_{2}\right)} \mathbf{u}_{l b},
\end{gathered}
$$

where it is noted that the $Y$-periodic constraint on the equilibrium equation is fulfilled with $\mathbf{k}=(0,0)^{T}$. The constraints will be imposed by use of the transformation method. Accordingly, a transformation matrix $\mathbf{T}$ is constructed, which links the full and reduced basis:

$$
\begin{gathered}
\mathbf{u}=\mathbf{T}(\mathbf{k}) \mathbf{u}_{\mathbf{k}}, \\
\left\{\begin{array}{c}
\mathbf{u}_{l} \\
\mathbf{u}_{b} \\
\mathbf{u}_{l b} \\
\mathbf{u}_{i} \\
\mathbf{u}_{r} \\
\mathbf{u}_{t} \\
\mathbf{u}_{b r} \\
\mathbf{u}_{l t} \\
\mathbf{u}_{r t}
\end{array}\right\}=\left[\begin{array}{cccc}
\mathbf{I} & \mathbf{0} & \mathbf{0} & \mathbf{0} \\
\mathbf{0} & \mathbf{I} & \mathbf{0} & \mathbf{0} \\
\mathbf{0} & \mathbf{0} & \mathbf{I} & \mathbf{0} \\
\mathbf{0} & \mathbf{0} & \mathbf{0} & \mathbf{I} \\
\mathbf{I} e^{i \mathbf{k}^{T} \mathbf{R}_{1}} & \mathbf{0} & \mathbf{0} & \mathbf{0} \\
\mathbf{0} & \mathbf{I} e^{i \mathbf{k}^{T} \mathbf{R}_{2}} & \mathbf{0} & \mathbf{0} \\
\mathbf{0} & \mathbf{0} & \mathbf{I} e^{i \mathbf{k}^{T} \mathbf{R}_{1}} & \mathbf{0} \\
\mathbf{0} & \mathbf{0} & \mathbf{I} e^{i \mathbf{k}^{T} \mathbf{R}_{2}} & \mathbf{0} \\
\mathbf{0} & \mathbf{0} & \mathbf{I} e^{i \mathbf{k}^{T}\left(\mathbf{R}_{1}+\mathbf{R}_{2}\right)} & \mathbf{0}
\end{array}\right]\left\{\begin{array}{c}
\mathbf{u}_{l} \\
\mathbf{u}_{b} \\
\mathbf{u}_{l b} \\
\mathbf{u}_{i}
\end{array}\right\},
\end{gathered}
$$

where subscript $\square_{\mathbf{k}}$ denotes representation in the $\mathbf{k}$ reduced basis. The reduced equilibrium equations (7) and the instability equation (10) are then obtained by substituting with the transformations $\chi^{j}=\mathbf{T}(\mathbf{0}) \chi_{\mathbf{0}}^{j}, \boldsymbol{\phi}=\mathbf{T}(\mathbf{k}) \boldsymbol{\phi}_{\mathbf{k}}$ and subsequent pre-multiplication by $\mathbf{T}^{H}$ :

$$
\begin{array}{rlrl}
\mathbf{K}_{\mathbf{0}} \chi_{\mathbf{0}}^{j} & =\mathbf{f}_{\mathbf{0}}^{j}, & \mathbf{K}_{\mathbf{0}}=\mathbf{T}(\mathbf{0})^{H} \mathbf{K T}(\mathbf{0}), \quad \mathbf{f}_{\mathbf{0}}^{j}=\mathbf{T}(\mathbf{0})^{H} \mathbf{f}^{j}, \\
\left(\mathbf{K}_{\mathbf{k}}-P \mathbf{G}_{\mathbf{k}}\right) \boldsymbol{\phi}_{\mathbf{k}}=\mathbf{0}, & \mathbf{K}_{\mathbf{k}}=\mathbf{T}(\mathbf{k})^{H} \mathbf{K T}(\mathbf{k}), \quad \mathbf{G}_{\mathbf{k}}=\mathbf{T}(\mathbf{k})^{H} \mathbf{G T}(\mathbf{k}),
\end{array}
$$

where the subscript $\square_{\mathbf{0}}$ implies reduction in basis $\mathbf{k}=\mathbf{0}$, and the superscript $\square^{H}$ denotes the hermitian transpose.

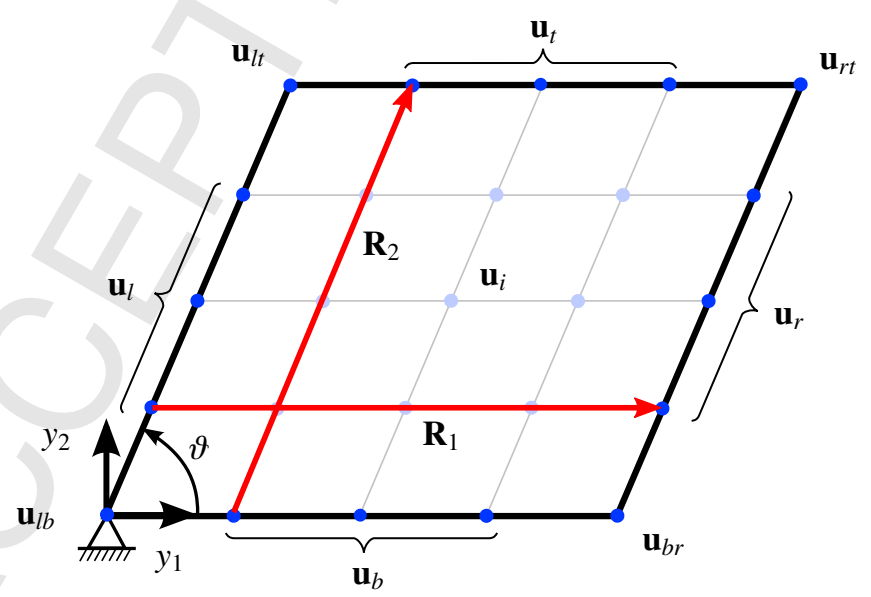

Figure 3: Discretized unit cell (rhombic for $\vartheta=60^{\circ}$, square for $\vartheta=90^{\circ}$ ) with indication of node indexing and lattice vectors $\mathbf{R}_{j}$. 
(a)

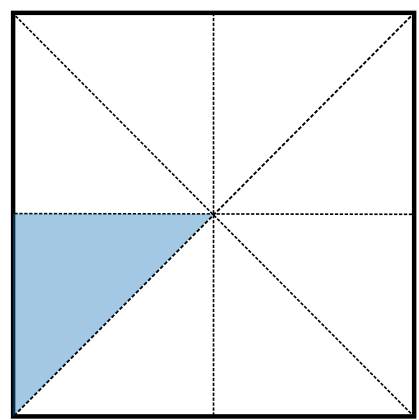

(b)

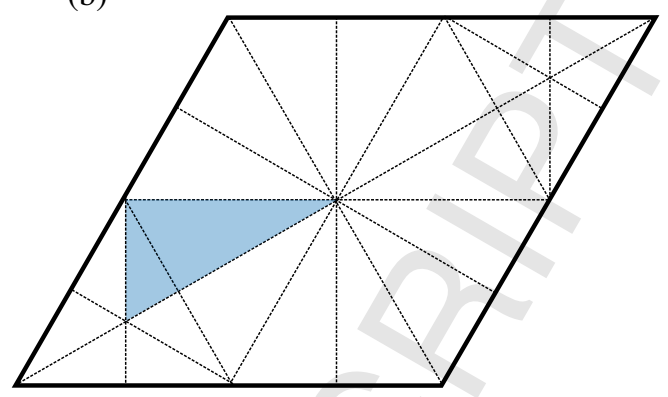

Figure 4: Symmetry lines and indication of the fundamental domain which comprise the design space for (a) square unit cell with $45^{\circ}$-symmetry, and (b) rhombic unit cell with $60^{\circ}$-symmetry.

\section{Topology optimization}

In this section, an optimization problem is formulated to design unit cell topologies with maximized strength against microscopic buckling instability for prescribed macroscopic load conditions. This includes a parametrization of the design, a problem formulation and a sensitivity analysis to enable gradient-based optimization.

\subsection{Design parametrization}

The problem is parametrized using the density-based approach, where each finite element within the unit cell is assigned with a relative element density $\rho_{e} \in[0,1]$. By this method, the constitutive properties $\mathbf{E}_{e}$ are interpolated between a void and solid phase using a modified SIMP-scheme [25]:

$$
\mathbf{E}_{e}= \begin{cases}\left(\rho_{\min }+\left(1-\rho_{\min }\right) \rho_{e}^{p}\right) \mathbf{E}_{0} & \text { for } \mathbf{K}, \mathbf{f}^{j} \\ \rho_{e}^{p} \mathbf{E}_{0} & \text { for } \mathbf{G}\end{cases}
$$

where $p$ is a penalization factor, $\rho_{\min }$ is the relative density of the void phase which is set to a small value to avoid numerical singularities, and $\mathbf{E}_{0}$ is the constitutive tensor of the solid phase. The modified scheme is used to eliminate the notoriously known problem of artificial modes which contaminate low density regions. To ensure that the scheme is successful, the value of $\rho_{\min }$ must be sufficiently large to stabilize low density regions while still being small enough to have negligible influence on the bifurcation loads. Through multiple numerical studies, $\rho_{\min }=10^{-4}$ was found to yield the best results without causing significant alterations of the loads.

In the specification of the design domain, symmetry constraints in the material distribution are utilized as illustrated in Figure 4. These geometric restrictions will enforce material symmetries in the material properties (e.g. square symmetry or isotropy, see [46]), but also substantially lower the computational cost of the stability analysis as it reduces the unique part of the Brillouin zone. Accordingly, element densities within the fundamental domain will be designated as design variables $\boldsymbol{\mu}$, and element densities of the complete domain $\boldsymbol{\rho}$ are obtained through the mapping:

$$
\rho=\mathbf{L} \mu
$$

where $\mathbf{L}$ is a mapping matrix with dimension $N \times n$.

\subsection{Problem formulation}

The pure strength optimization problem is defined as maximization of the most critical bifurcation load of all modes spanned within the Brillouin zone $\mathscr{B}$. This may be formulated as a min-max problem of the inverse bifurcation 
load factors $\lambda_{i}(\mathbf{k})=P_{i}(\mathbf{k})^{-1}$ for wavevectors $\mathbf{k} \in \mathscr{B}$ and macroscopic stress $\overline{\boldsymbol{\sigma}}$ :

$$
\begin{array}{ll}
\min _{\boldsymbol{\mu}}: & \max _{\mathbf{k} \in \mathscr{B}} \max _{i=1, . . I} \lambda_{i}(\mathbf{k}) \\
\text { s.t. : } & \left(\mathbf{G}_{\mathbf{k}}(\overline{\boldsymbol{\sigma}})-\lambda_{i} \mathbf{K}_{\mathbf{k}}\right) \boldsymbol{\phi}_{i \mathbf{k}}=\mathbf{0}, \quad i=1, . ., I, \quad \mathbf{k} \in \mathscr{B} \\
& \mathbf{K}_{\mathbf{0}} \chi_{\mathbf{0}}^{j}=\mathbf{f}_{\mathbf{0}}^{j}, \quad j=1,2,3 \\
& \frac{1}{|Y|} \sum_{e=1}^{N} \rho_{e} v_{e} \leq V^{*} \\
& 0 \leq \mu_{e} \leq 1, \quad e=1, \ldots, n
\end{array}
$$

where the $I$ most critical modes are included as candidates. The constraints are constituted by the state equations of the homogenization and instability problems, a volume fraction constraint with upper bound $V^{*}$ computed by finite element volumes $v_{e}$, and box constraints on the design variables.

In practice, the optimization is implemented as a bound formulation [25] to avoid lack of smoothness if multiple states coalesce. This involves a discretization of the wavevector space spanning the Brillouin zone into $J$ optimization points whereafter the $K$ most critical eigenvalues $\lambda_{i}\left(\mathbf{k}_{j}\right)$ of the discrete set $i=1, \ldots, I, j=1, . ., J$ are required to be smaller than a bound variable which becomes the new minimization objective. The optimization points should ideally be placed along the boundary of the irreducible Brillouin zone and will thus be highly case dependent. More specific details on proper placement for various load states and geometric symmetries are presented in Section 5. Furthermore, to ensure smooth convergence of the optimization algorithm, the number of eigenvalues $K$ and bands $I$ should be chosen such that all modes within the most critical range are included in the formulation, but they should not be excessively large as it comes at increased computational cost. As the number of modes entering the critical range gradually increases during optimization, an ideal selection strategy of $K$ and $I$ would thus involve an adaptive scheme where the values are updated continuously. In this work, however, we utilize a more pragmatic approach with constant values $K=25$ and $I=10$ to avoid risking convergence for increased efficiency. These values are selected based on experience and the choice is obviously case dependent.

\subsection{Sensitivity analysis}

Computation of the bifurcation load sensitivities is a complex task as the eigenvalues may have multiplicity which introduces lack of differentiability and thus requires special treatment. Here, a generalized gradient method based on perturbation analysis is utilized which involves solving a series of sub-optimization problems [37]. To retain clarity, only the case of distinct eigenvalues are presented here, while the case with multiplicity is included in Appendix A.

The sensitivity of the inverse bifurcation load factor $\lambda=P^{-1}$ can be computed with the adjoint method by [47]:

$$
\frac{d \lambda}{d \rho_{e}}=\boldsymbol{\phi}^{H}\left(\frac{\partial \mathbf{G}}{\partial \rho_{e}}+\frac{\partial \mathbf{G}}{\partial \overline{\boldsymbol{\varepsilon}}} \frac{d \overline{\boldsymbol{\varepsilon}}}{d \rho_{e}}-\lambda \frac{\partial \mathbf{K}}{\partial \rho_{e}}\right) \boldsymbol{\phi}+\sum_{j=1}^{3}\left(\mathbf{T}(\mathbf{0}) \mathbf{v}^{j}\right)^{H}\left(\frac{\partial \mathbf{K}}{\partial \rho_{e}} \chi^{j}-\frac{\partial \mathbf{f}^{j}}{\partial \rho_{e}}\right)
$$

expressed with the reconstructed mode $\boldsymbol{\phi}=\mathbf{T}(\mathbf{k}) \boldsymbol{\phi}_{\mathbf{k}}$ and test-fields $\chi^{j}=\mathbf{T}(\mathbf{0}) \chi_{\mathbf{0}}^{j}$ to enable element decomposition, and where the mode has been $\mathbf{K}$-orthonormalized such that $\boldsymbol{\phi}^{H} \mathbf{K} \boldsymbol{\phi}=1$. The adjoint variables $\mathbf{v}^{j}$ are found as solutions to:

$$
\mathbf{K}_{\mathbf{0}} \mathbf{v}^{j}=-\mathbf{T}(\mathbf{0})^{H}\left(\boldsymbol{\phi}^{H} \frac{\partial \mathbf{G}}{\partial \chi^{j}} \boldsymbol{\phi}\right)^{H}, \quad j=1,2,3
$$

Evaluation of the adjoint loads will be performed on element level followed by assembly to global form. In expanded format, this process can be described by:

$$
\boldsymbol{\phi}^{H} \frac{\partial \mathbf{G}}{\partial \chi^{j}} \boldsymbol{\phi}=\sum_{e=1}^{N}\left\{\boldsymbol{\phi}_{e}^{H} \frac{\partial \mathbf{G}_{e}}{\partial \chi_{e, 1}^{j}} \boldsymbol{\phi}_{e}, \cdots, \boldsymbol{\phi}_{e}^{H} \frac{\partial \mathbf{G}_{e}}{\partial \chi_{e, n n}^{j}} \boldsymbol{\phi}_{e}\right\}^{T},
$$

where:

$$
\frac{\partial \mathbf{G}_{e}}{\partial \chi_{e, i}^{j}}=\int_{Y_{e}} \frac{\partial \mathbf{N}^{T}}{\partial y_{l}} \frac{\partial\left(\sigma_{l m}\right)_{e}}{\partial \chi_{e, i}^{j}} \frac{\partial \mathbf{N}}{\partial y_{m}} d Y, \quad \frac{\partial \sigma_{e}}{\partial \chi_{e, i}^{j}}=-\mathbf{E}_{e} \mathbf{B}_{e} \mathbf{e}_{i j} \overline{\boldsymbol{\varepsilon}},
$$


where $n n$ denotes the number of degrees of freedom of element $e$, and $\mathbf{e}_{i j}$ is a $8 \times 3$ single entry matrix with 1 at element $(i, j)$.

The term $\frac{\partial \mathbf{G}}{\partial \bar{\varepsilon}} \frac{d \bar{\varepsilon}}{d \rho_{e}}$ accounting for variation in homogenized strain will vanish in the load case of prescribed strain where $\overline{\boldsymbol{\varepsilon}}$ is independent of the homogenized constitutive properties. For prescribed stress where $\overline{\boldsymbol{\varepsilon}}=\overline{\mathbf{E}}^{-1} \overline{\boldsymbol{\sigma}}$, the sensitivity of the homogenized strain $d \bar{\varepsilon} / d \rho_{e}$ is obtained by solving:

$$
\overline{\mathbf{E}} \frac{d \overline{\boldsymbol{\varepsilon}}}{d \rho_{e}}=-\frac{d \overline{\mathbf{E}}}{d \rho_{e}} \overline{\boldsymbol{\varepsilon}}
$$

where the sensitivity of the homogenized constitutive matrix is:

$$
\frac{d \bar{E}_{i j}}{d \rho_{e}}=\frac{1}{|Y|} \int_{Y_{e}}\left(\tilde{\boldsymbol{\varepsilon}}^{i}-\mathbf{B}_{e} \chi_{e}^{i}\right)^{T} \frac{d \mathbf{E}_{e}}{d \rho_{e}}\left(\tilde{\boldsymbol{\varepsilon}}^{j}-\mathbf{B}_{e} \chi_{e}^{j}\right) d Y,
$$

with $\frac{d \mathbf{E}_{e}}{d \rho_{e}}=p\left(1-\rho_{\min }\right) \rho_{e}^{p-1} \mathbf{E}_{0}$. The first part of the term is obtained by:

$$
\frac{\partial \mathbf{G}}{\partial \bar{\varepsilon}_{k}}=\sum_{e=1}^{N} \int_{Y_{e}} \frac{\partial \mathbf{N}^{T}}{\partial y_{l}} \frac{\partial\left(\sigma_{l m}\right)_{e}}{\partial \bar{\varepsilon}_{k}} \frac{\partial \mathbf{N}}{\partial y_{m}} d Y, \quad \frac{\partial \boldsymbol{\sigma}_{e}}{\partial \bar{\varepsilon}_{k}}=\mathbf{E}_{e}\left(\mathbf{I}-\mathbf{B}_{e} \boldsymbol{X}_{e}\right) \mathbf{e}_{k}
$$

where $\mathbf{e}_{k}$ is a $3 \times 1$ single entry vector with 1 at element $k$.

The derivatives of the global stiffness matrices $\mathbf{K}, \mathbf{G}$ and loads $\mathbf{f}^{j}$ are found using equations (8), (11) and (17):

$$
\frac{\partial \mathbf{K}}{\partial \rho_{e}}=p\left(1-\rho_{\min }\right) \rho_{e}^{p-1} \mathbf{K}_{e}^{0}, \quad \frac{\partial \mathbf{f}^{j}}{\partial \rho_{e}}=p\left(1-\rho_{\min }\right) \rho_{e}^{p-1} \mathbf{f}_{e}^{0^{j}}, \quad \frac{\partial \mathbf{G}}{\partial \rho_{e}}=p \rho_{e}^{p-1} \mathbf{G}_{e}^{0},
$$

where $\mathbf{K}_{e}^{0}, \mathbf{G}_{e}^{0}, \mathbf{f}_{e}^{0^{j}}$ are element matrices and vectors evaluated with the constitutive matrix of the solid phase $\mathbf{E}_{0}$.

\section{Computational model}

A schematic of the computational model which is used to solve the optimization problem is visualized in Figure 5. The model utilizes a standard topology optimization procedure where regularization is performed with a Heaviside projection scheme, see Appendix B for more details. The optimization procedure is an iterative process in which incremental design updates are performed based on gradient information until convergence is achieved. Within each iteration, design variables are first mapped to the full structure whereafter the filtering is applied to obtain physical element densities $\hat{\boldsymbol{\rho}}$. Next, the finite element model is employed to compute the objective and constraint functions and their sensitivities w.r.t. the physical densities. For the objective function, this involves solving the homogenization problem and instability problem to obtain the $K$ most critical eigenvalues $\lambda_{i}\left(\mathbf{k}_{j}\right),\{i, j\} \in S$ and associated generalized gradients $d \Lambda_{k m}^{i j} / d \hat{\boldsymbol{\rho}}$ (see end of Section 3.2. for a discussion on how to choose $K$ and $I$ ). Subsequently, the eigenvalue sensitivities w.r.t. the design variables are obtained by computing unfiltered generalized gradients with the chain rule, remapping them to the design field and solving a set of sub-optimization problems. Finally, the design update is computed using the Method of Moving Asymptotes [48], and if the change is below a specified threshold, the process is terminated.

\section{Results}

In this section, the computational model is utilized to design strength optimized microstructures for the three compressive macroscopic stress states of biaxial, uniaxial and shear loading. The optimizations are performed with offset in three different basic microstructures, including the square, triangular and hexagonal honeycomb for various geometric symmetry constraints. Subsequently, all designs are subject to a performance comparison of their strength and stiffness characteristics, which involve computation of failure surfaces in macroscopic stress space as well as their elastic properties. It is noted that extensive numerical testing has been performed with other initial material 
distributions to investigate how the optimization procedure is influenced by the initial guess, but in all cases the results either converged towards similar microstructure designs or designs with worse performance.

The simulations are conducted for unit cells with size $d=1$, discretized by $200 \times 200$ elements, a base material with Young's modulus $E_{0}=1$ and Poisson's ratio $v=0.3$, and modelled under the plane stress assumption. The optimizations are constrained with a maximum volume fraction $V^{*}=0.3$, and regularized using a penalization factor $p=3.0$, density filter radius $R=0.03 d$, Heaviside projection threshold $\eta=0.5$, and Heaviside intensity factor $\beta=1$ with the continuation scheme $\beta \rightarrow 2 \beta$ updated every 100 iterations and a maximum value of $\beta_{\max }=256$.

Topology optimization

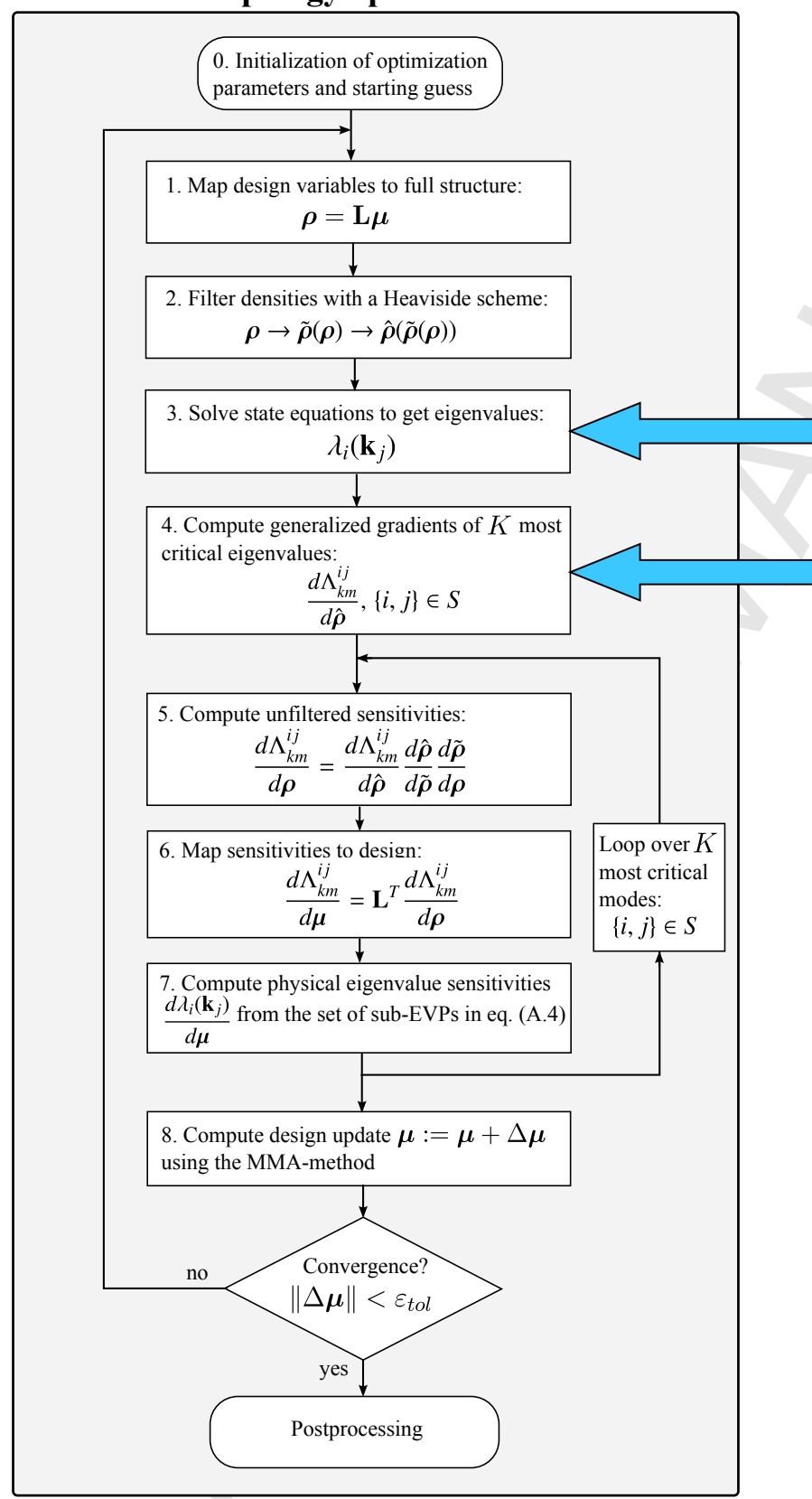

\section{Finite element analysis}

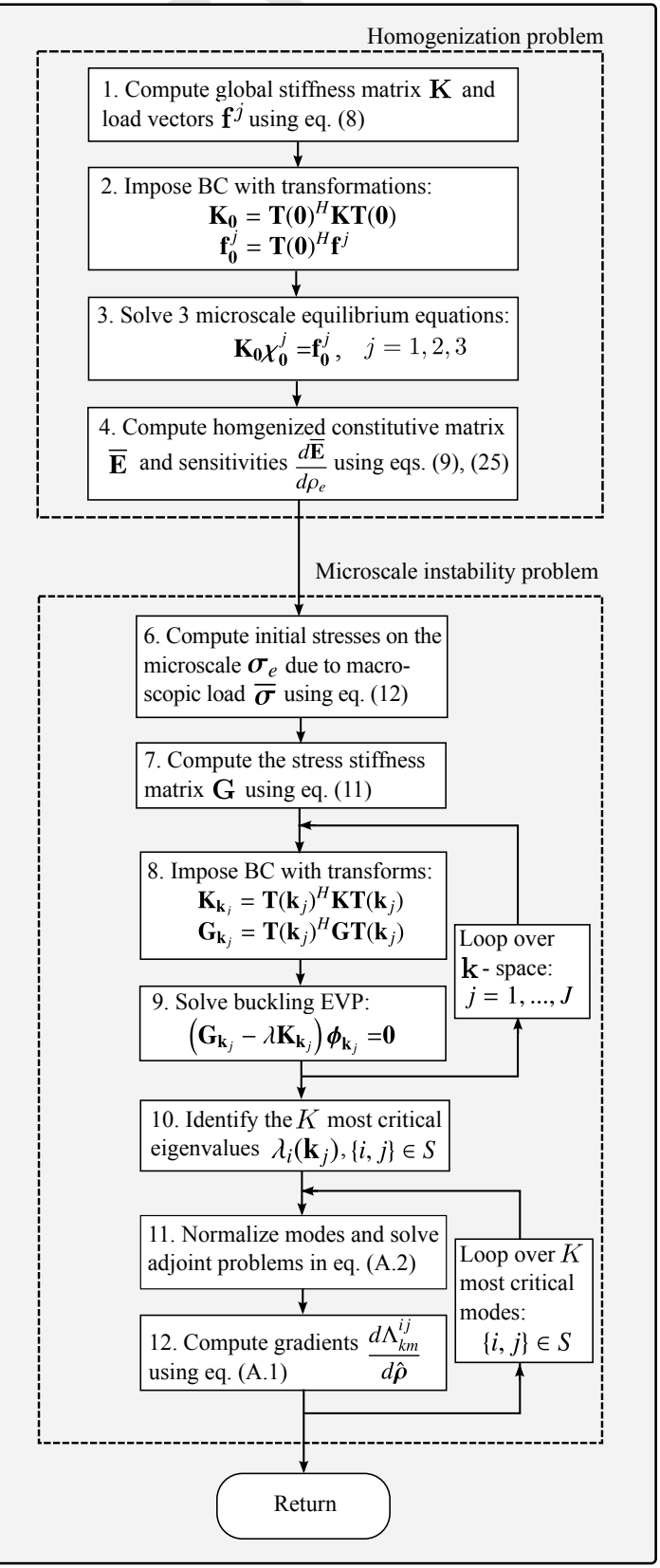

Figure 5: Computational model of the optimization problem. 


\subsection{Optimization for the biaxial load case}

The first example considers a biaxial compressive load case where $\bar{\sigma}=(-1,-1,0)^{T}$. The designs are constrained with $45^{\circ}$ and $60^{\circ}$-symmetries on the square and rhombic unit cells, respectively, and the instability problem is discretized into 7 points along the boundary of the irreducible Brillouin zone as shown in Figure 6. The optimization points are placed at the major symmetry points where the local minima usually occurs, and one near $\Gamma$ to capture the long wavelength failure mode. After the optimization has been performed, a detailed stability analysis with higher resolution is then conducted to assess the quality of the optimized designs. The results are summarized in Figures 7-9, showcasing the initial and optimized topologies along with the band diagrams of the 10 first bifurcation loads on the boundary of the irreducible Brillouin zone.

For all three cases, the optimized designs turn out to be first-order hierarchical type structures which offer substantial improvements in buckling strength compared to the initial zero-order designs. Their superior strength characteristics can be attributed to the hierarchical material distributions which provide increased effective bending stiffness and hence buckling resistance of the structural members. The degree of optimality of the optimized designs is visualized through the band diagrams. For all three cases, the bands exhibit minor variations and are tightly stacked, thus implying that the microstructures have made near optimal use of available material in order to strengthen the different modes of failure equally. In contrast, the bands of the initial designs display large variation and spacing, indicating that a single failure mode is dominating and that the microstructures are non-optimal in terms of strength.

To facilitate a more detailed performance assessment of the initial and optimized designs, a quantitative comparison of strength under biaxial loading is shown in Table 1, while the general strength characteristics under arbitrary loading are visualized by failure surfaces in Figure 10a. Under the design load, the optimized (non-isotropic) square microstructure attains the highest strength with $P / E_{0}=0.00901$, corresponding to a relative improvement of $344 \%$ over its initial solid form. The optimized triangle is nearly as strong as the square with $P / E_{0}=0.00900$, but offers the largest relative improvement of all three cases with an increase of $379 \%$. The worst performance is achieved for the optimized hexagon with a strength of $P / E_{0}=0.00828$ despite the fact that its basic form has far superior strength under biaxial loading. The reduced gains can be attributed to the fact that it has the shortest beam members of the three cases, and hence benefits less from the hierarchical configuration. Considering their strength under general nonsymmetric loading, the failure surfaces illustrate that the optimized designs offer profound strength improvements for a majority of the investigated load-range. For all cases, the largest performance improvement is achieved at the design load, while the gains gradually diminish for increasingly non-symmetric loading. In the vicinity of the design load, the best retention of strength is achieved with the optimized triangle closely followed by the square and hexagon. However, in terms of general robustness towards arbitrary loading the square is regarded superior as the triangle and hexagon exhibit high susceptibility towards uniaxial and mixed loading.

While the optimized topologies provide an increase in strength, it comes at the cost of reductions in stiffness. This is indicated through a qualitative comparison of the designs on the effective Young's modulus and bulk modulus in
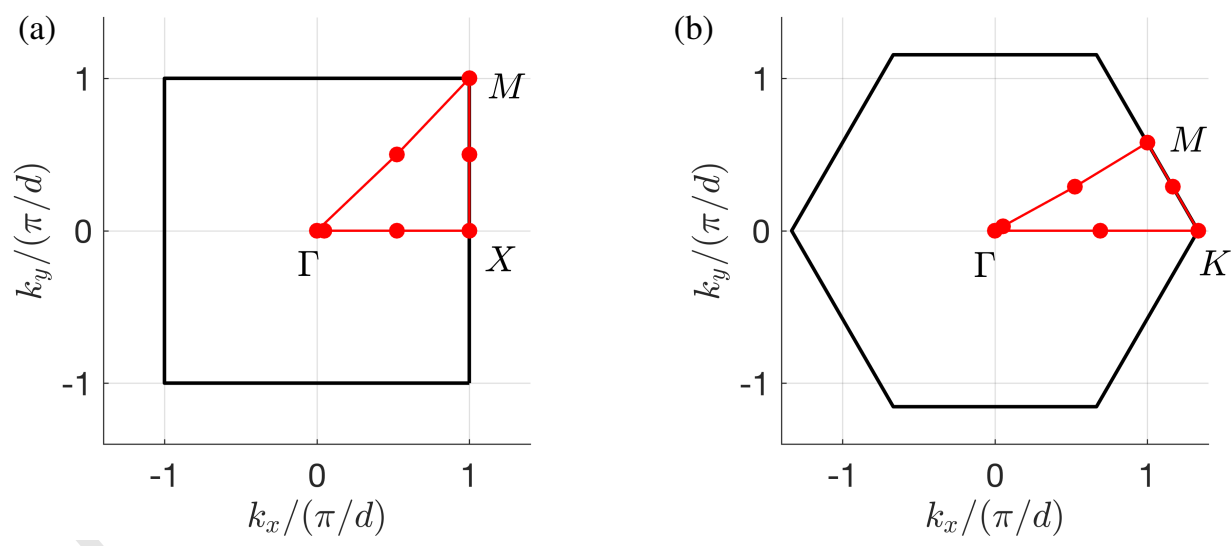

Figure 6: Optimization points on the boundary of the irreducible Brillouin zone under symmetric loading for (a) $45^{\circ}$-symmetric square unit cell, and (b) $60^{\circ}$-symmetric rhombic unit cell. 


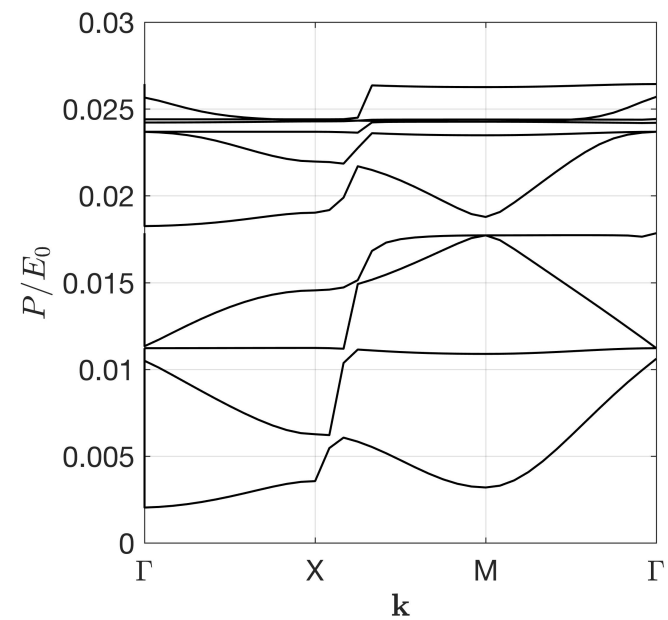

(a) Initial band diagram

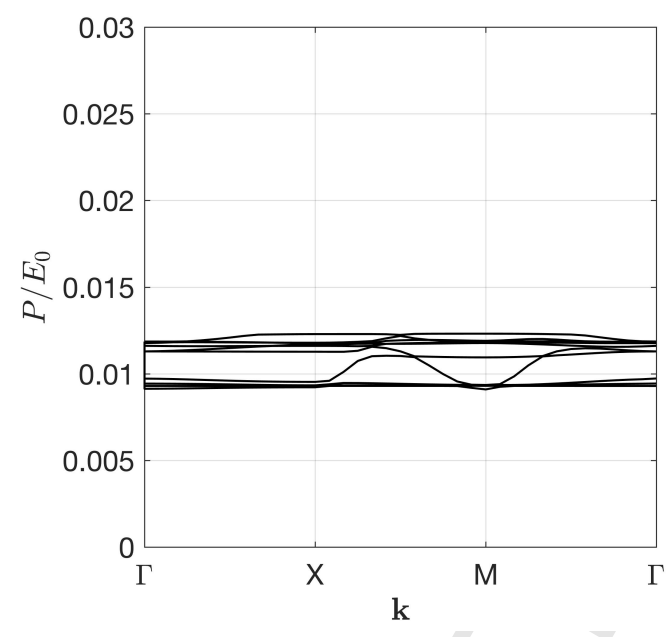

(c) Optimized band diagram

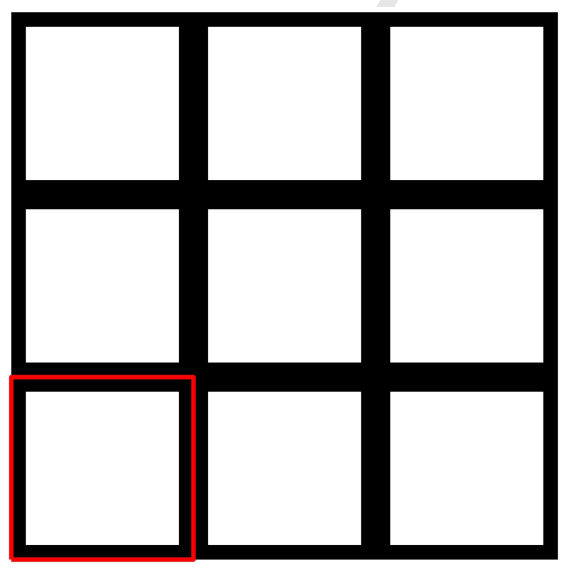

(b) Initial topology

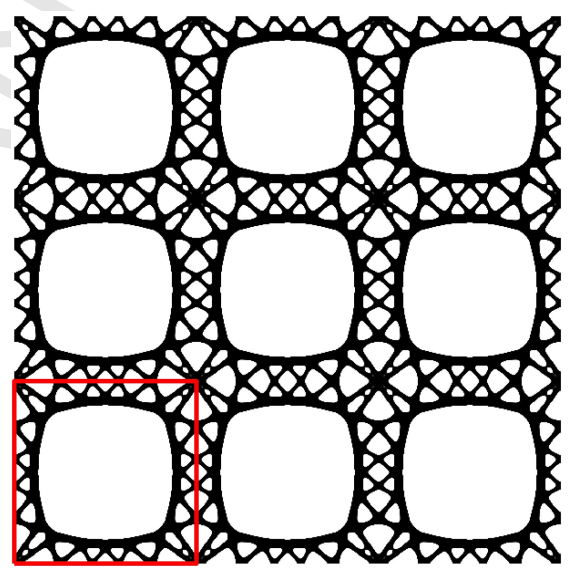

(d) Optimized topology

Figure 7: Optimization of the square microstructure for the load $\bar{\sigma}=(-1,-1,0)^{T}$. (b), (d) show a $3 \times 3$ super-cell of the initial and optimized topology, and (a), (c) show the band diagram of the 10 most critical bifurcation loads along the boundary of the irreducible Brillouin zone.

Table 1, and with the directional Young's modulus in Figure 10b. The reductions in stiffness are a direct consequence of the hierarchical structures, which are not stiffness optimal when loaded in compression. Under macroscopic biaxial loading, this condition prevails in all designs and as a result, modest reductions in the bulk modulus can be observed, ranging within $17-26 \%$ compared to the initial designs. The largest bulk stiffness is achieved with the optimized square while the hexagon performs most poorly, and their bulk modulus range within 49-54\% of the upper theoretical Hashin-Shtrikman bound. Under macroscopic uniaxial loading, more diverse stiffness characteristics can be observed. In this case, the triangle remains compression-dominated and consequently, the optimized hierarchical design yields a reduction of $42 \%$ in the Young's modulus. Conversely, the optimized hexagon experiences a stiffness increase of $70 \%$ in the Young's modulus due to the increased bending stiffness of the hierarchical beams. As a result, it even becomes stronger than the optimized triangle whose solid form is far superior. The square microstructure looses $44 \%$ stiffness in uniaxial loading applied in the direction of orthotropy where it is purely compression-dominated. However, as offaligned directions involve bending, the hierarchical structure provides a remarkable stiffness increase of $440 \%$ in the 


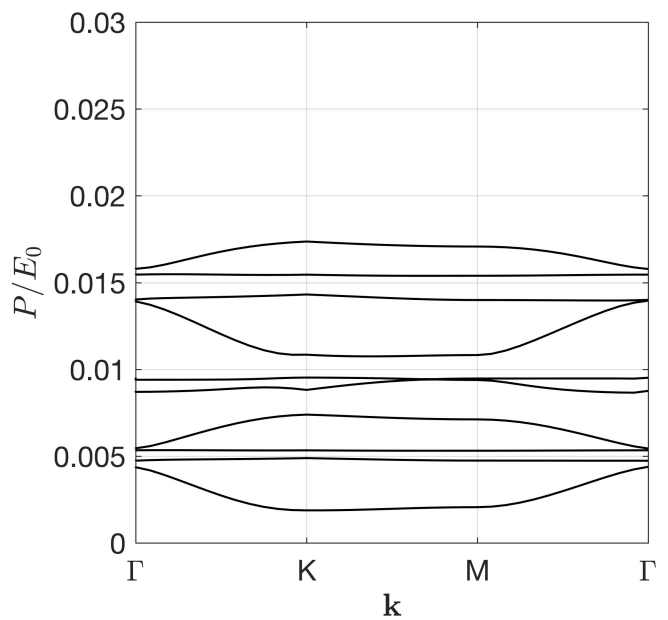

(a) Initial band diagram

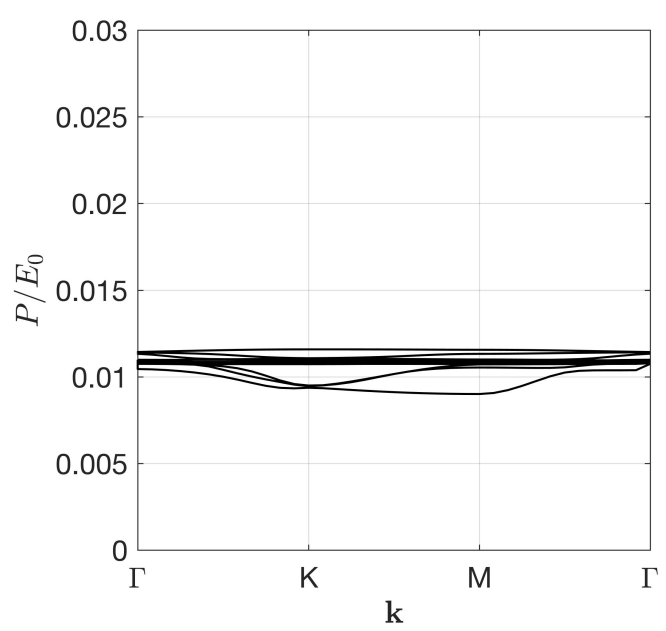

(c) Optimized band diagram

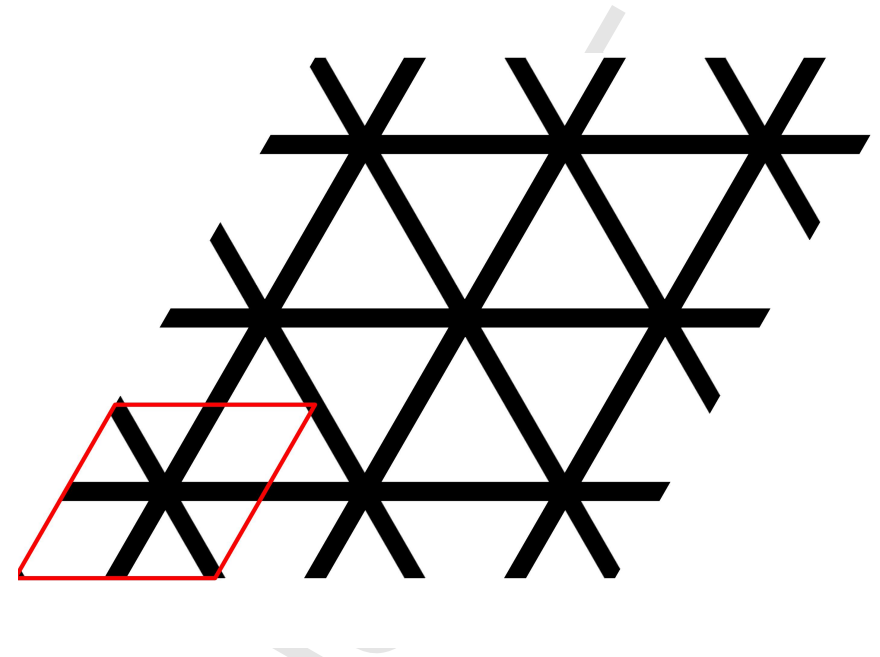

(b) Initial topology

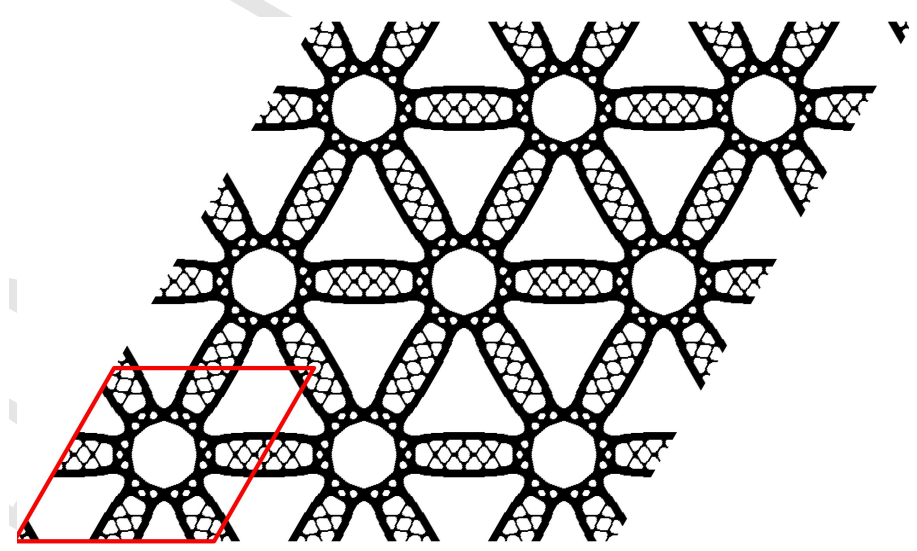

(d) Optimized topology

Figure 8: Optimization of the triangular microstructure for the load $\bar{\sigma}=(-1,-1,0)^{T}$. (b), (d) show a $3 \times 3$ super-cell of the initial and optimized topology, and (a), (c) show the band diagram of the 10 most critical bifurcation loads along the boundary of the irreducible Brillouin zone.

$45^{\circ}$-orientation of maximal bending, which consequently leads to a significant reduction in its degree of anisotropy. Consequently, the Young's modulus of the optimized square almost gets on par with that of the isotropic optimized triangle and hexagon in its worst orientation, while it surpasses them substantially when loaded in the directions of orthotropy.

\subsection{Optimization of the uniaxial load case}

The second example considers optimization of the uniaxial compressive load case where $\bar{\sigma}=(-1,0,0)^{T}$. Here, an extended number of optimization points in $\mathbf{k}$-space are utilized, as non-symmetric loading disrupts some of the symmetry properties which prevailed in the biaxial load case, see Figure 11. The optimization is conducted for the cases with $45^{\circ}$ and $60^{\circ}$-symmetry constraints and initial topologies as previously, and in addition for the case with $90^{\circ}$-symmetry in the square unit cell. The optimized topologies are shown in Figure 12, while the failure surfaces and effective Young's modulus are visualized in Figure 13. 


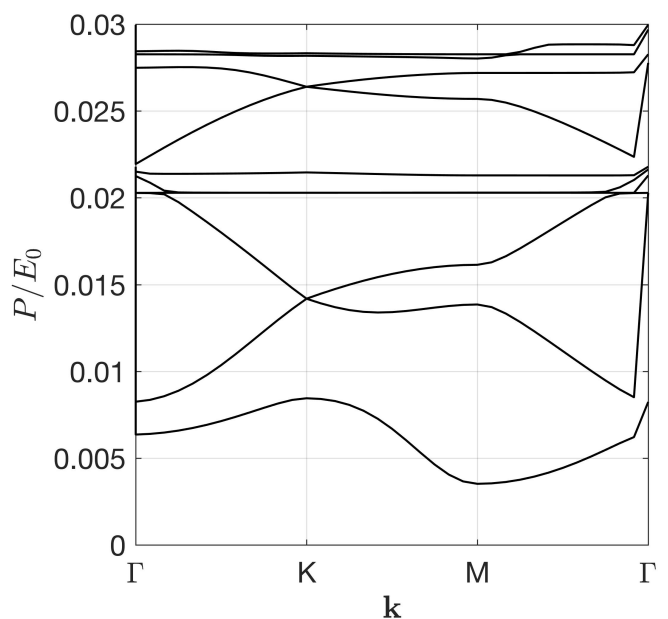

(a) Initial band diagram

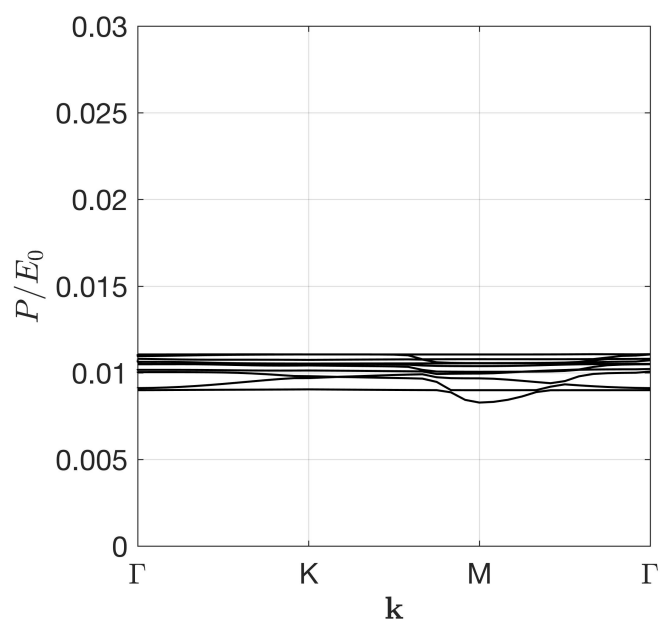

(c) Optimized band diagram

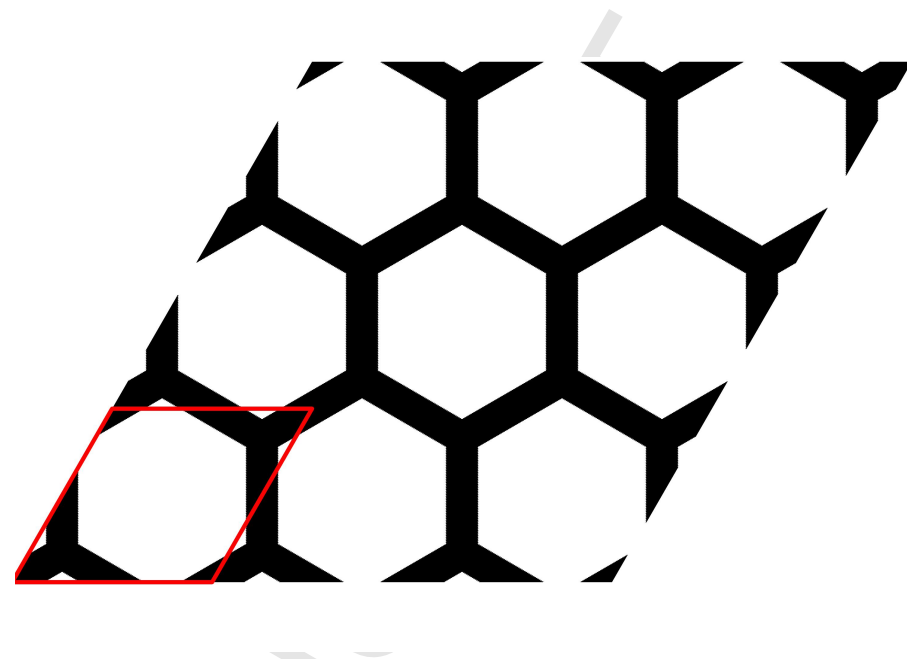

(b) Initial topology

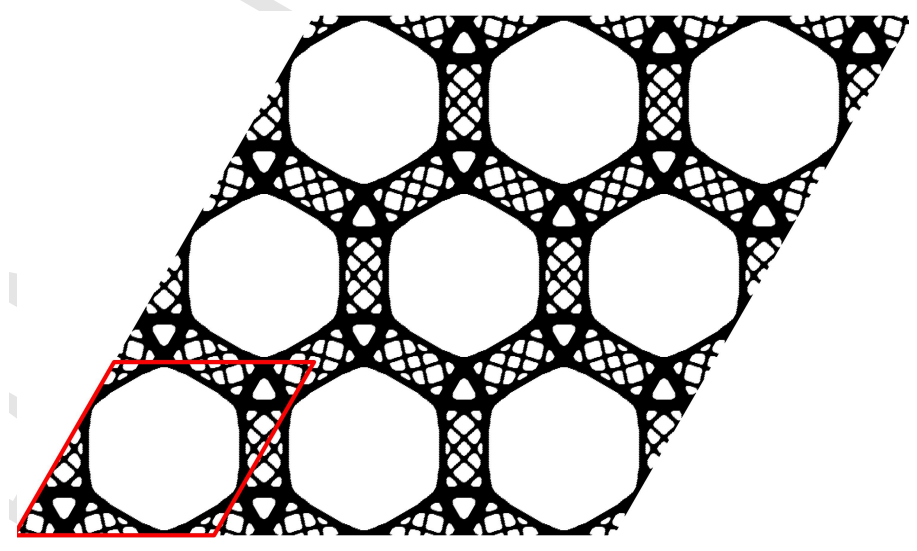

(d) Optimized topology

Figure 9: Optimization of the hexagonal microstructure for the load $\bar{\sigma}=(-1,-1,0)^{T}$. (b), (d) show a $3 \times 3$ super-cell of the initial and optimized topology, and (a), (c) show the band diagram of the 10 most critical bifurcation loads along the boundary of the irreducible Brillouin zone.

\begin{tabular}{|c|c|c|c|c|c|c|c|c|c|}
\hline \multirow{2}{*}{ Topology } & \multicolumn{3}{|c|}{ Buckling strength } & \multicolumn{3}{|c|}{ Bulk modulus } & \multicolumn{3}{|c|}{ Young's modulus $\left(\bar{E}_{1}\right)$} \\
\hline & $P_{\text {init }} / E_{0}$ & $P_{o p t} / E_{0}$ & $P_{\text {opt }} / P_{\text {init }}$ & $\bar{\kappa}_{\text {init }} / E_{0}$ & $\overline{\kappa_{o p t}} / E_{0}$ & $\bar{\kappa}_{\text {opt }} / \bar{\kappa}_{\text {init }}$ & $\bar{E}_{\text {init }} / E_{0}$ & $\bar{E}_{o p t} / E_{0}$ & $\bar{E}_{\text {opt }} / \bar{E}_{\text {init }}$ \\
\hline Square & 0.00203 & 0.00901 & 4.44 & 0.0872 & 0.0724 & 0.83 & 0.1639 & 0.0922 & 0.56 \\
\hline Hexagon & 0.00352 & 0.00828 & 2.35 & 0.0880 & 0.0656 & 0.74 & 0.0421 & 0.0715 & 1.70 \\
\hline Triangle & 0.00188 & 0.00900 & 4.79 & 0.0876 & 0.0670 & 0.76 & 0.1172 & 0.0675 & 0.58 \\
\hline
\end{tabular}

Table 1: Buckling strength under biaxial loading and elastic properties of the initial structures and structures optimized for biaxial loading. The upper Hashin-Shtrikman bounds of the Young's modulus are: $E_{i s o}^{*} / E_{0}=0.1250$ (hexagon and triangle), $E_{s q}^{*} / E_{0}=0.1765$ (square in the orthotropy directions), and for the Bulk modulus $\kappa_{i s o}^{*} / E_{0}=\kappa_{s q}^{*} / E_{0}=0.0932$.

The results show that the optimized square topology with $90^{\circ}$-symmetry has the highest strength for the design load of pure uniaxial compression. The topology consists of thick beams aligned with the load direction which 
(a)

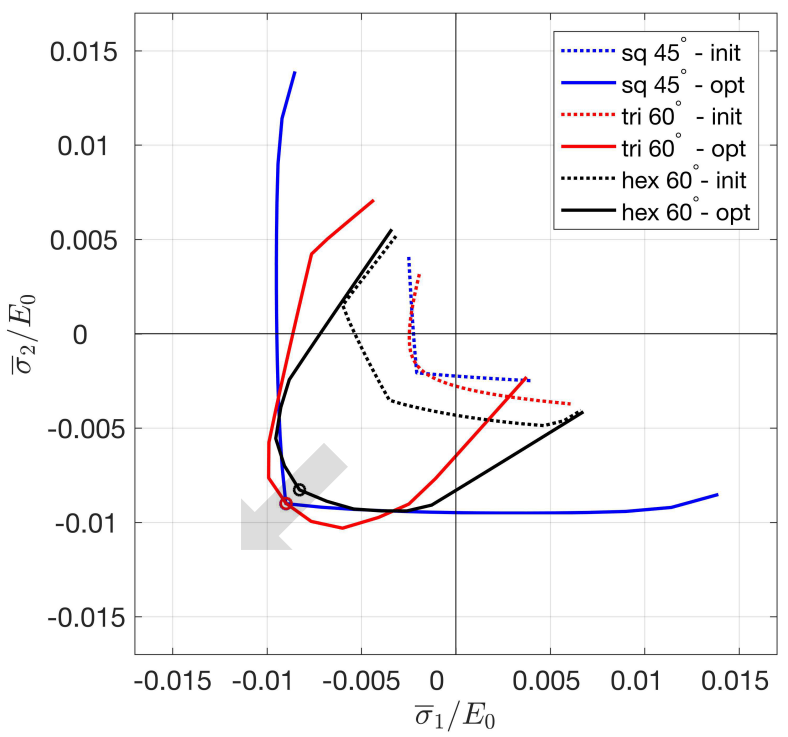

(b)

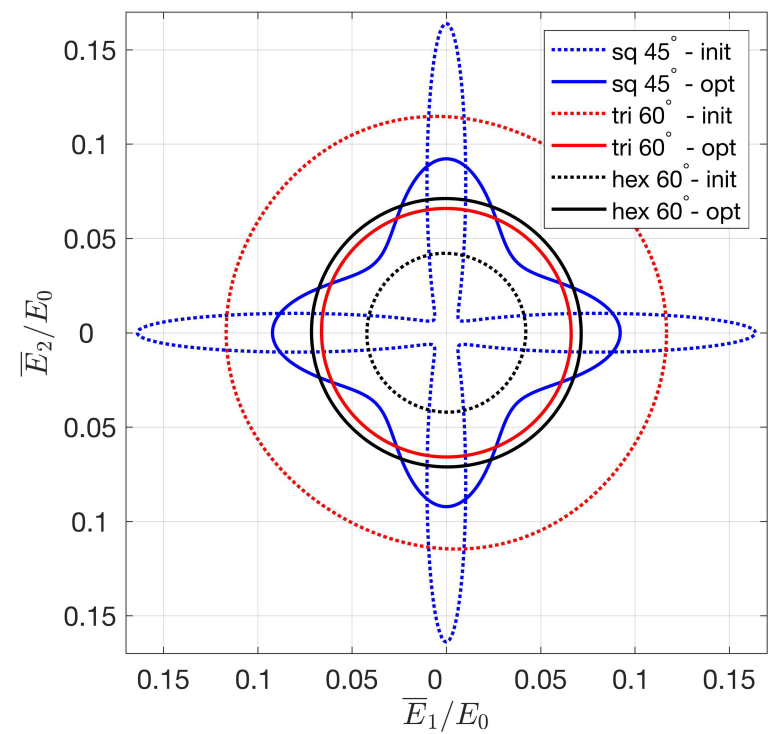

Figure 10: Buckling strength and stiffness characteristics of the initial designs and designs optimized for the biaxial load $\bar{\sigma}=(-1,-1,0)^{T}$, represented by (a) their failure surface in macroscopic stress space, and (b) their effective Young's modulus.

(a)

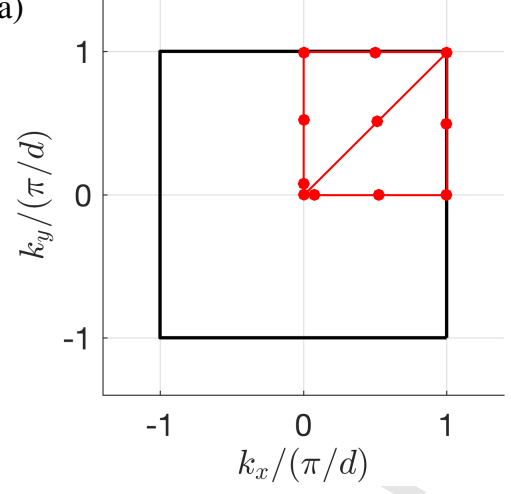

(b)

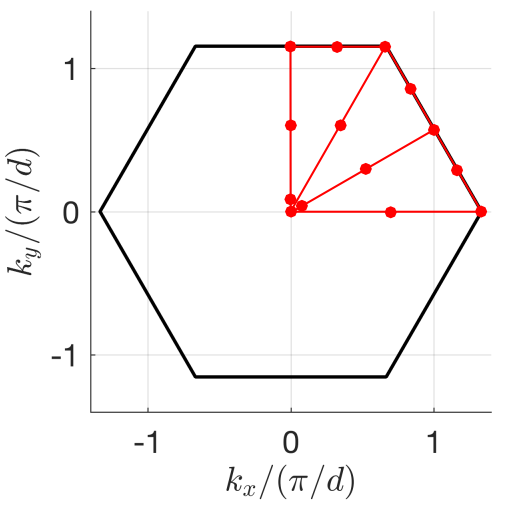

Figure 11: Optimization points in the Brillouin zone in the non-symmetric uniaxial load case for (a) square unit cell and (b) rhombic unit cell.

have a hierarchical structure to maximize the effective bending stiffness, and thinner interconnecting beams to stiffen against long wavelength instability. The superior strength can be attributed to the fact that the relaxed symmetry constraints do not couple its material distribution in the load direction to any transverse direction. Hence it utilizes all material optimally to stiffen against the exact uniaxial load-condition, and as a result obtains more than $30.6 \%$ higher strength compared to any of the other designs. Although the increased freedom associated with lessened symmetry enables a higher degree of anisotropy and increased strength potential, it comes at the cost of reductions in general robustness towards alternate load conditions which are not included in the optimization formulation. Specifically, the $90^{\circ}$-topology has essentially no strength and low stiffness under compression in the $y_{2}$-direction. Conversely, the $45^{\circ}$ and $60^{\circ}$-topologies offer an increased robustness as the symmetry constraints couple the material distribution to other directions, but it comes at the cost of reduced strength and stiffness under the design load. 


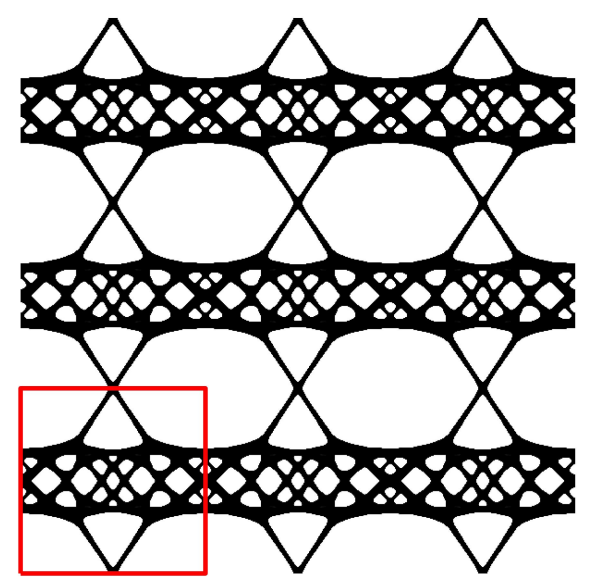

(a) Square, $90^{\circ}$-symmetry

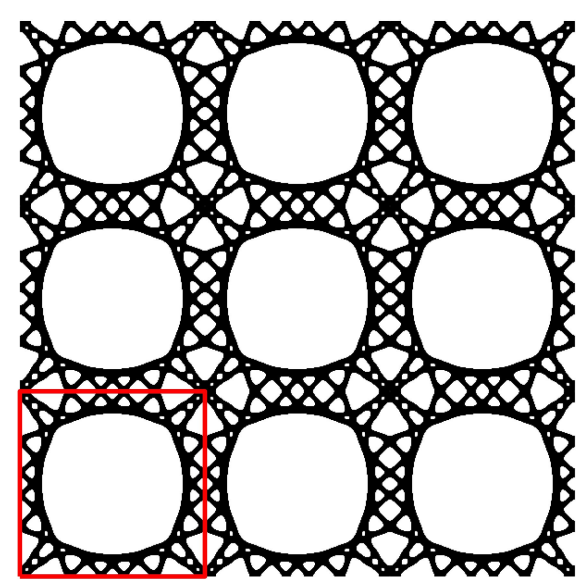

(b) Square, $45^{\circ}$-symmetry

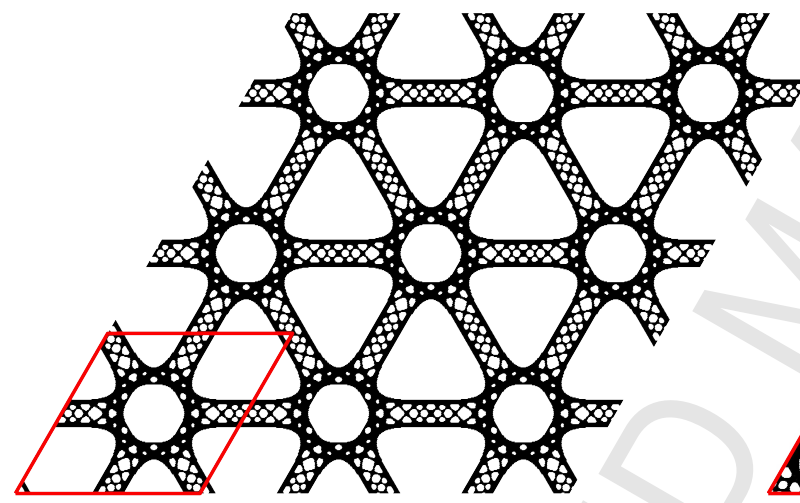

(c) Triangle, $60^{\circ}$-symmetry

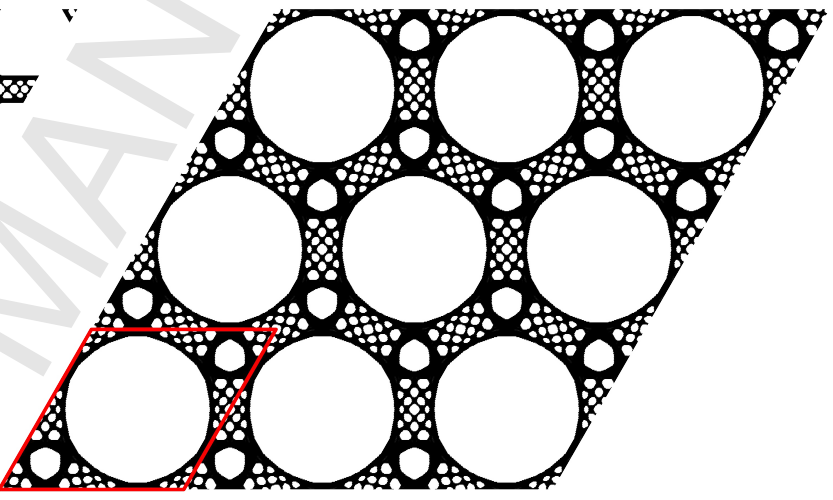

(d) Hexagon, $60^{\circ}$-symmetry

Figure 12: $3 \times 3$ super-cell of the topologies optimized for the uniaxial load case, $\bar{\sigma}=(-1,0,0)^{T}$.

\subsection{Optimization of the mixed load case}

The final example considers the load case of combined compression and tension, $\bar{\sigma}=(-1,1,0)^{T}$, which correspond to pure shear when the material is oriented $45^{\circ}$ to the frame of reference. The optimization is conducted with the same design points as for uniaxial loading, and the stability analysis is restricted to search only for positive eigenvalues to avoid optimization of the reversed load case. The optimized topologies are shown in Figure 14, while associated failure surfaces and the effective shear modulus are illustrated in Figure 15.

In this case, the failure surfaces clearly visualize that the optimized designs attain stability under compression through transverse tensile loading. The physical explanation to this behaviour resides within the geometrical configuration of the designs. Specifically, tension is exploited to restrict rotation and decrease the effective length of the beam constituents, which thus leads to an increase in buckling strength. For the $90^{\circ}$-topology, the increased rotational stiffness is achieved with thin tension bars spaced far apart, whereas the $45^{\circ}$ and $60^{\circ}$-topologies obtain it through an increased effective beam width at the points of connection. A direct consequence of the rotational stiffening is that the most critical failure modes involve sway-like deformation of the beams. As this deformation mode has zero curvature at the center-points of the beams, the necessity for large bending stiffness in this region through a hierarchical structure is lost, hence explaining why the beams narrow in.

Comparing the performance of the optimized designs, the highest strength is achieved for the $90^{\circ}$-topology yield- 
(a)

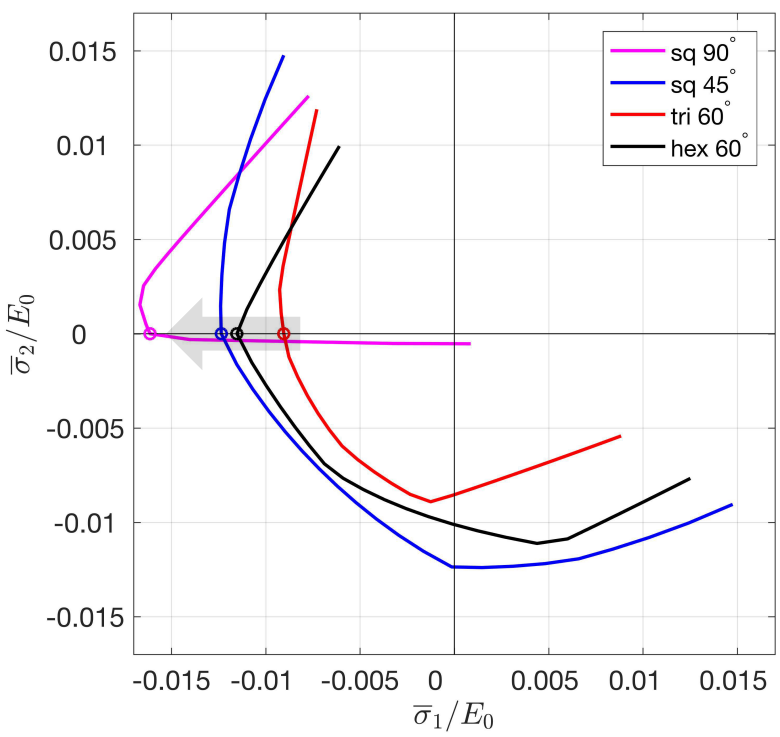

(b)

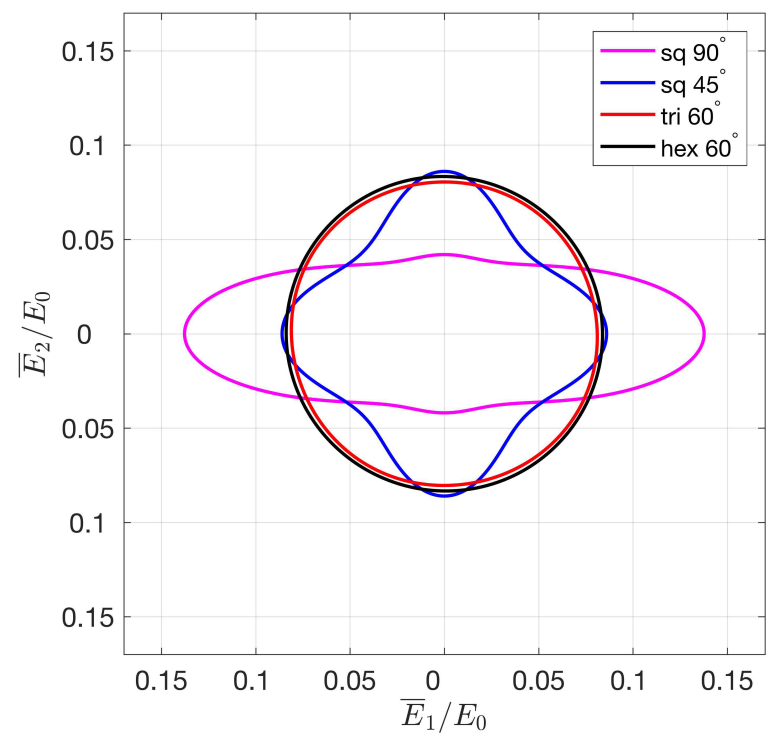

Figure 13: Buckling strength and stiffness characteristics of the designs optimized for the uniaxial load $\bar{\sigma}=(-1,0,0)^{T}$, represented by (a) their failure surface in macroscopic stress space, and (b) their effective Young's modulus.

ing more than a $36.1 \%$ increase in strength compared to any of the other designs. However, with superior strength comes the added cost of high reliance on tension to remain stable, resulting in non-existing load carrying capability under uniaxial or biaxial loading. In terms of stiffness, the $90^{\circ}$-topology exhibits very high degree of anisotropy, and its maximal shear modulus in the $45^{\circ}$ orientation is significantly smaller than any of the other designs. Accordingly, the added robustness of the $45^{\circ}$ and $60^{\circ}$-topologies will be preferable in most cases over the increased strength potential of the $90^{\circ}$-topology.

\subsection{Overall performance comparison}

A comparison of the failure surfaces of all optimized designs is shown in Figure 16. The figure clearly visualizes the framework's capability to tailor microstructures with optimized strength for different load conditions, and how geometric symmetries are exploitable to control the shape of the failure surfaces and obtain various degrees of robustness. Comparing the performance of the designs with regards to the different optimization directions, it appears that the topologies optimized for the mixed load case realize the highest strength potential at the design load, but they also have the worst performance in terms of universal robustness, as they realize their strength through a reliance on transverse loading. In contrast, the topologies optimized for the biaxial and uniaxial case have little to none of such dependency and instead achieve high directional strength through the geometric symmetry constraints. Although the strength of these designs is not as high at the design load, they may however still be preferred in applications where the load condition varies or is not fully known. Comparing the strength of the optimized designs across the different choices of initial design and symmetry constraints, the $90^{\circ}$-square topologies are found to be substantially superior at the exact design load but lack in robustness. The $45^{\circ}$-square and hexagonal topologies perform almost equally in all load cases at the design load, whereas the triangular topologies in most optimization cases achieve the lowest strength. Nonetheless, the triangular topologies offer high robustness which is on par with that of the $45^{\circ}$-square, whereas the hexagonal topologies are substantially more susceptible towards varying load conditions.

Comparing the optimized designs to the initial designs, it is important to highlight that the major improvements in strength come at the cost of reductions in stiffness. For example, the stiffnesses of the initial designs all range within $93-95 \%$ of the theoretical bulk modulus bound while both the triangular and square design (in their directions of orthotropy) also range within $93-94 \%$ of the Young's modulus bound, c.f. Table 1. After the designs have been optimized for maximized buckling strength, their stiffnesses are however reduced to just 50-70\% of the theoretical 


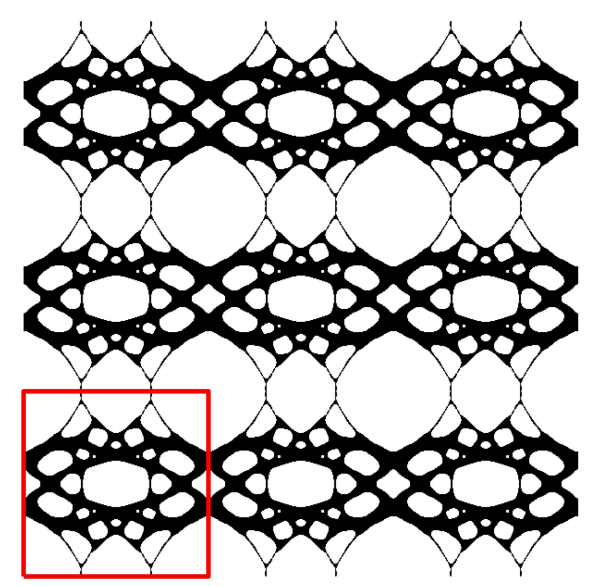

(a) Square, $90^{\circ}$-symmetry

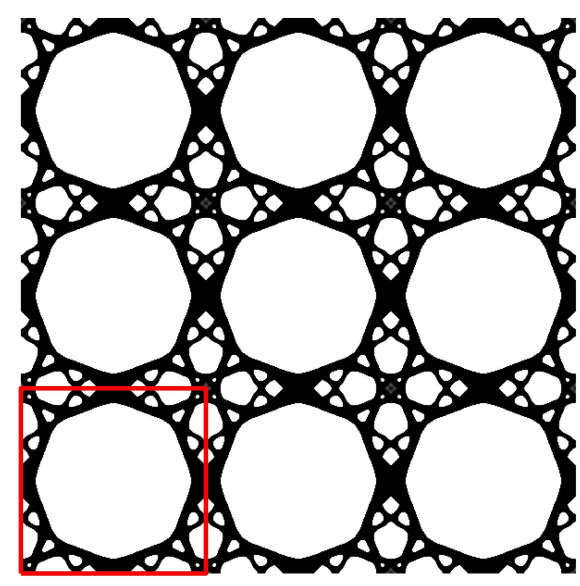

(b) Square, $45^{\circ}$-symmetry

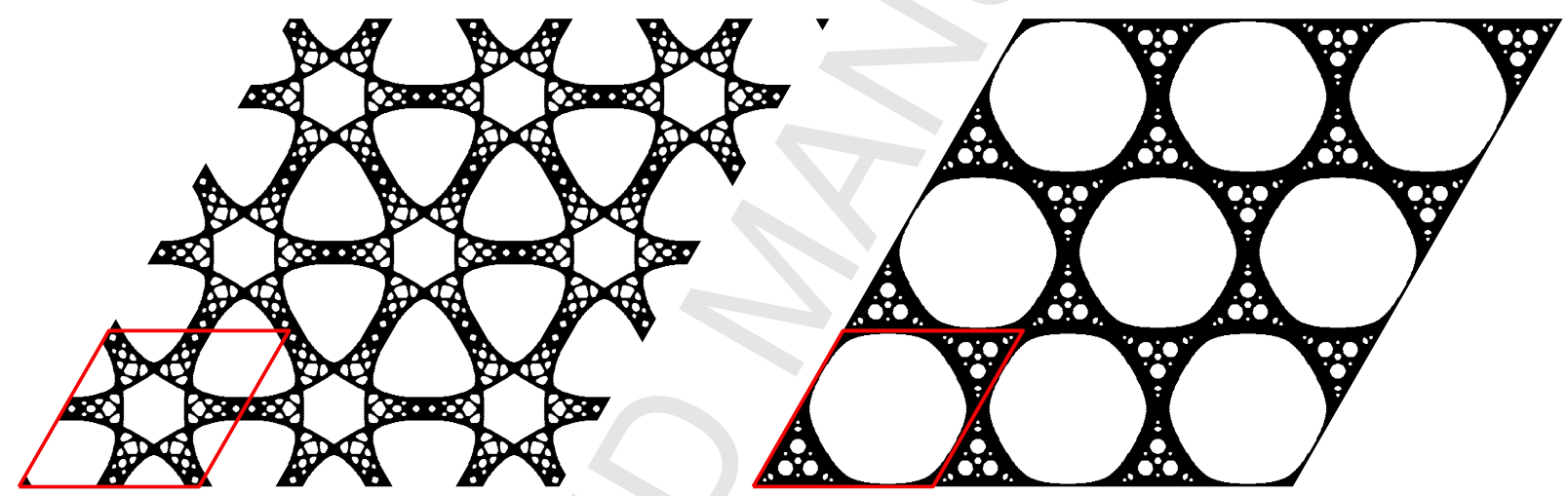

(c) Triangle, $60^{\circ}$-symmetry

(d) Hexagon, $60^{\circ}$-symmetry

Figure 14: $3 \times 3$ super-cell of the topologies optimized for the mixed load case, $\bar{\sigma}=(-1,1,0)^{T}$.

bounds. This trade-off could be explored further through Pareto studies by gradually varying the weight between stiffness and strength objectives, but this goes beyond the scope of the paper.

Lastly, it is noted that the geometry of the optimized designs exhibit an appreciable resemblance across the different load directions despite the prominent differences in failure surfaces. This characteristic will be very advantageous in the extended multi-scale design problem, as it enables more seamless interface connections when different microstructures are combined into a macroscopic composite.

\section{Concluding remarks}

This work has presented a topology optimization framework for designing microstructures of periodic materials with optimized strength against initiation of microscopic buckling instability. The basis is a simplified linear stability model which utilizes homogenization theory and Bloch-wave analysis to predict instability in the microscale for specified macroscopic stress states, and a topology optimization problem which is formulated to improve the buckling strength through redistribution of material in the unit cell.

The model has been employed to optimize three simple 2D microstructures subject to various macroscopic compressive load conditions. In all cases, the optimized designs contained hierarchical microstructures which offered 
(a)

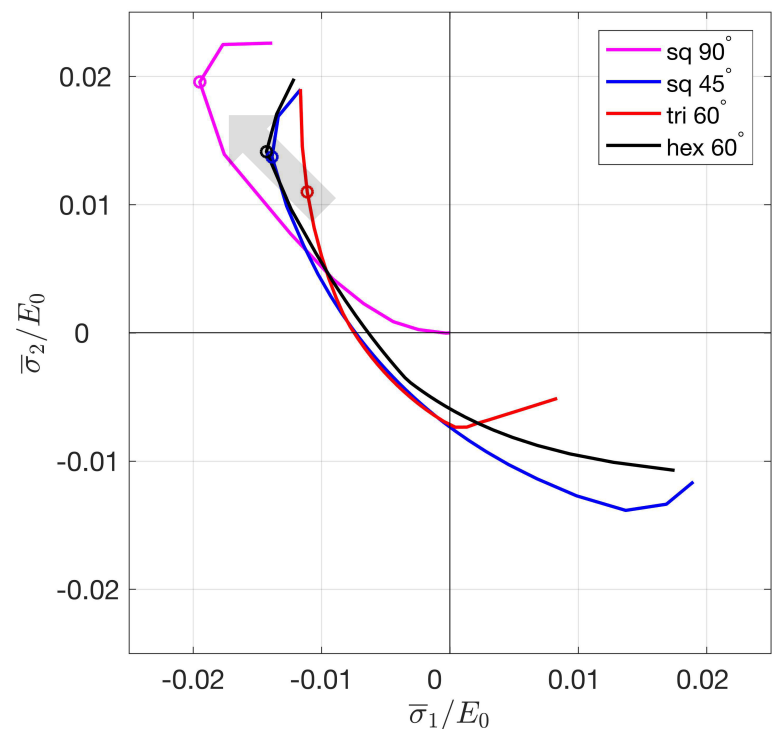

(b)

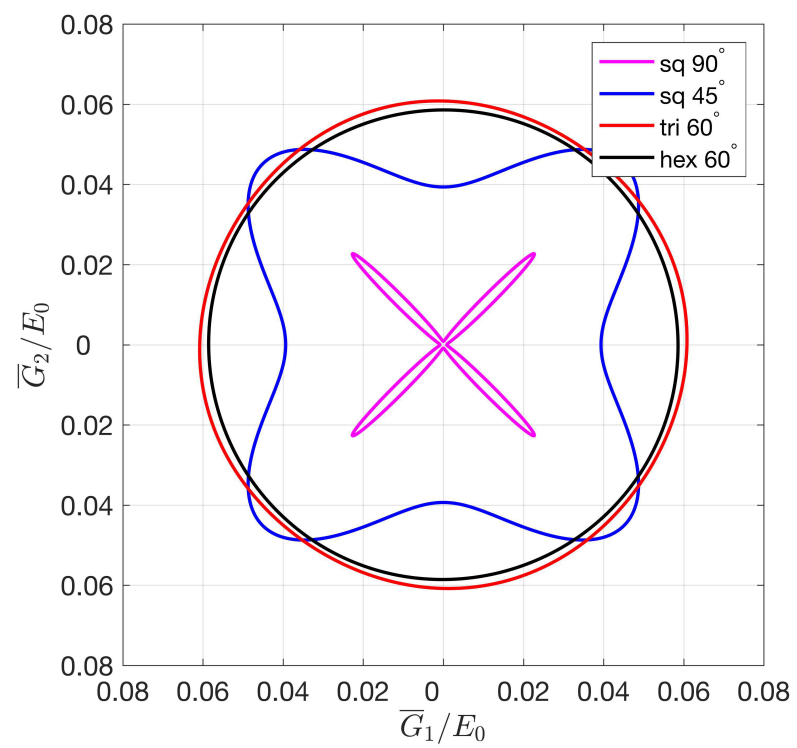

Figure 15: Buckling strength and stiffness characteristics of the designs optimized for the mixed load $\bar{\sigma}=(-1,1,0)^{T}$, represented by (a) their failure surface in macroscopic stress space, and (b) their effective shear modulus.

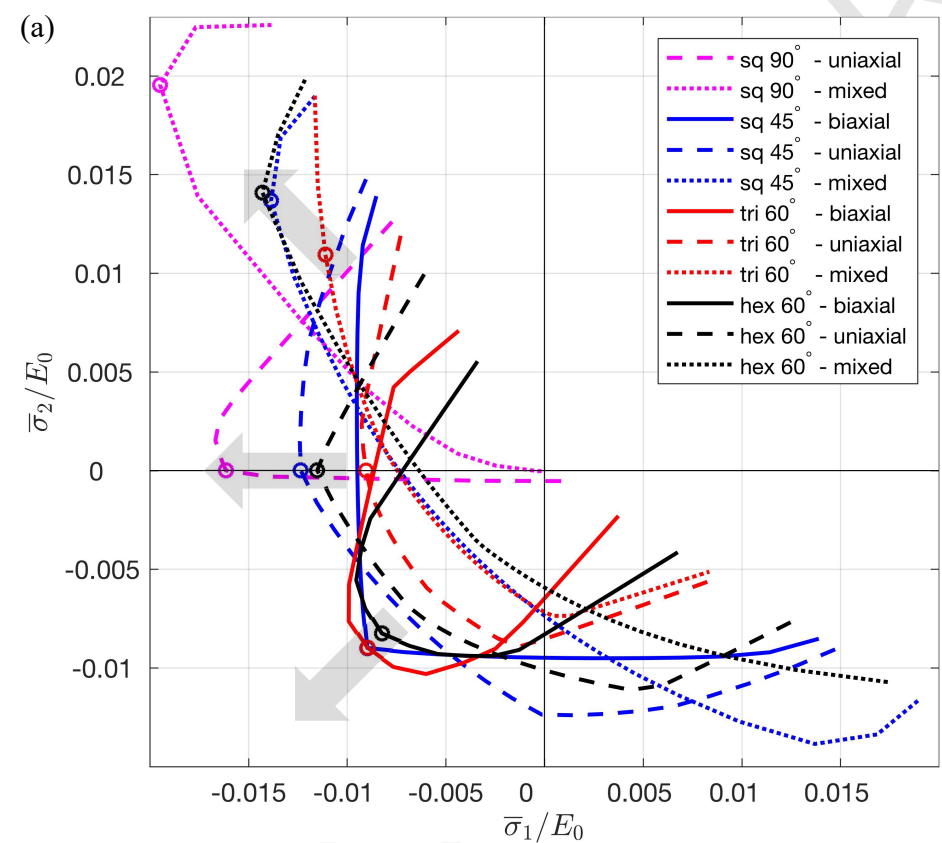

(b)
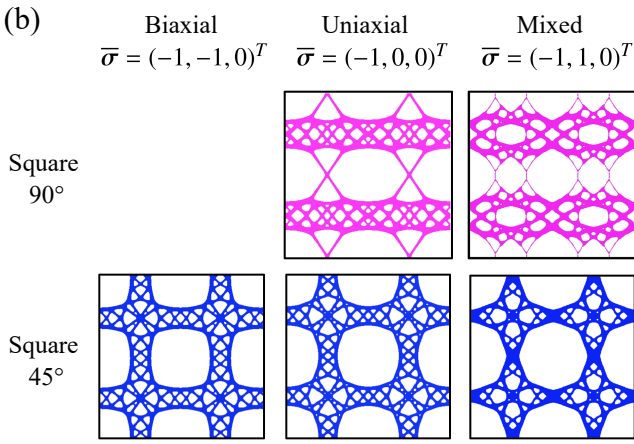

Figure 16: (a) Comparison of failure surfaces optimized for 3 different load directions and various symmetry constraints/initial designs, and (b) corresponding optimized topologies.

profound improvements in strength compared to the initial designs. However, a majority of the designs also suffered from significant reductions in stiffness since the originally tension/compression dominated solid beams had to be converted to stiffness suboptimal hierarchical ones in order to increase stiffness for bending dominated buckling modes. In general, it was demonstrated how the framework functions as an excellent tool for tailoring desired strength characteristics by control of the prescribed load conditions and geometric symmetry constraints within the material 
distribution of the unit cell. It was found that utilization of few geometric symmetries yields the highest strength potential at the design load but also reduces overall load robustness, whereas the opposite effect is achieved with many symmetries.

The considered optimal design study resulted in structures with first order hierarchy. The reason higher orders did not show up can most probably be linked to the minimum length scale imposed by the enhanced buckling load and the design resolution and filter size at the very beginning of the optimization procedure. Accordingly, it is expected that considerably increased mesh resolution, lowering of the filter radius and also smaller volume fractions eventually will result in the appearance of further levels of hierarchy, thus yielding an improved buckling strength potential as discussed in e.g. [15] and [2]. Such studies and extensions are left for future work.

The current work is first of its kind to consider systematic optimization of microstructural buckling instability including local and non-local buckling modes. Nonetheless, it has several limitations that need to be addressed. A main point is the extension to more advanced buckling models, as linear elastic buckling is not always accurate in predicting true buckling modes, and hence should be substituted with a geometrically non-linear model. Even more importantly, the simplified model disregards any post-buckling behaviour, and since the presented structures have multiple critical buckling modes as a natural outcome of the optimization, it is likely that they exhibit a catastrophic post-buckling response. This undesirable phenomenon may potentially be avoided by a much more complex nonlinear model, or more simply by imposing the additional constraint that the second and higher bifurcation loads must be significantly larger than the most critical load. The latter approach of "band engineering" has already successfully been applied for problems related to photonics in [49]. Other important aspects for future work are experimental verification and extension to $3 \mathrm{D}$.

\section{Acknowledgements}

The authors acknowledge the support of the Villum Fonden through the Villum Investigator Project InnoTop. In addition, the author would like to thank Miguel M. Neves for valuable discussions regarding length scales and the validity of the governing equations, and thank Niels Aage for sharing his knowledge on the computational aspects of the problem.

\section{Appendix A. Sensitivity of eigenvalues with multiplicity}

When an eigenvalue is multiple, i.e. $\tilde{\lambda}=\lambda_{i}=\ldots=\lambda_{i+M-1}$ where $M$ is the multiplicity, the eigenmode will be a non-unique linear combination of the independent modes $\phi_{i}$. This introduces lack of differentiability, which complicates the sensitivity analysis. The problem is dealt with using a perturbation method as presented in [37], and involves solving a sub-eigenvalue problem for finding the $M$ physical sensitivities of the $M$-tuple eigenvalue for a given design variable perturbation. The method utilizes directional derivatives in conjunction with a sub-optimization problem for finding the best design-perturbation (direction of steepest descent). Here, a simplified version without a sub-optimization is used, where sensitivities are found by individual design variable perturbations. This approach is only approximative and may result in reduced convergence rates of the optimization, but it has reduced computational cost and is less complicated to implement.

Following the procedure of the simplified method applied to the inverse bifurcation load, generalized gradients $d \Lambda_{k m} / d \rho_{e}, k, m=1, . ., M$ are computed:

$$
\frac{d \Lambda_{k m}}{d \rho_{e}}=\boldsymbol{\phi}_{k}^{H}\left(\frac{\partial \mathbf{G}}{\partial \rho_{e}}+\frac{\partial \mathbf{G}}{\partial \overline{\boldsymbol{\varepsilon}}} \frac{d \overline{\boldsymbol{\varepsilon}}}{d \rho_{e}}-\tilde{\lambda} \frac{\partial \mathbf{K}}{\partial \rho_{e}}\right) \boldsymbol{\phi}_{m}+\sum_{j=1}^{3}\left(\mathbf{T}(\mathbf{0}) \mathbf{v}_{k m}^{j}\right)^{H}\left(\frac{\partial \mathbf{K}}{\partial \rho_{e}} \chi^{j}-\frac{\partial \mathbf{f}^{j}}{\partial \rho_{e}}\right)
$$

where the multipliers $\mathbf{v}_{k m}^{j}$ are solutions to the adjoint problems:

$$
\mathbf{K}_{\mathbf{0}} \mathbf{v}_{k m}^{j}=-\mathbf{T}(\mathbf{0})^{H}\left(\boldsymbol{\phi}_{k}^{H} \frac{\partial \mathbf{G}}{\partial \chi^{j}} \boldsymbol{\phi}_{m}\right)^{H}
$$


and where the independent modes $\boldsymbol{\phi}_{k}$ are assumed to be $\mathbf{K}$-orthogonalized, i.e. satisfy $\boldsymbol{\phi}_{k}^{H} \mathbf{K} \boldsymbol{\phi}_{m}=\delta_{k m}$. The gradients evaluated with respect to design variables $\mu$ are then obtained by the mapping:

$$
\frac{d \Lambda_{k m}}{d \boldsymbol{\mu}}=\mathbf{L}^{T} \frac{d \Lambda_{k m}}{d \boldsymbol{\rho}},
$$

and the sensitivities of the multiple eigenvalue are then found by solving the following set of sub-eigenvalue problems:

$$
\frac{d \tilde{\lambda}}{d \mu_{e}}=\left\{\gamma: \operatorname{det}\left(\frac{d \Lambda_{k m}}{d \mu_{e}}-\gamma \delta_{k m}\right)=0, \quad k, m=1, . ., M\right\}, \quad e=1, . ., n
$$

In the optimization, only the largest sensitivity of the $M$ eigenvalues for each element will used as these describe the 'worst case' design-change in a minimization problem. Furthermore, it is noted that the method reduces to the distinct eigenvalue method when $M=1$. Hence it is sufficient only to implement the generalized method without having to distinct between the cases.

\section{Appendix B. Regularization method}

The optimization problem is regularized with a density filter in conjunction with a Heaviside projection to eliminate mesh-dependency and avoid checker-board patters [50]. The density filtering of element $e$ is performed by averaging over all elements within a circle of radius $R$ :

$$
\tilde{\rho}_{e}=\frac{\sum_{j \in N_{e}} w\left(\mathbf{y}_{j}-\mathbf{y}_{e}\right) v_{j} \rho_{j}}{\sum_{j \in N_{e}} w\left(\mathbf{y}_{j}-\mathbf{y}_{e}\right) v_{j}}
$$

where $N_{e}=\left\{j \in 1, \ldots, N \mid R>\left\|\mathbf{y}_{j}-\mathbf{y}_{e}\right\|\right\}$ is an index-set of elements within the filter radius, $v_{j}$ is the volume of element $j$, and $w(\mathbf{y})=\max (0, R-\|\mathbf{y}\|)$ is a linear weight function. Subsequently, a smoothed Heaviside projection is applied to remove blurred edges following from the density filter:

$$
\bar{\rho}_{e}=\frac{\tanh (\beta \eta)+\tanh \left(\beta\left(\tilde{\rho_{e}}-\eta\right)\right)}{\tanh (\beta \eta)+\tanh (\beta(1-\eta))},
$$

where $\beta$ is a constant controlling the projection intensity, and $\eta$ is a projection threshold. This filtering technique will be employed in conjunction with a continuation strategy where $\beta$ is increased incrementally to ensure proper convergence towards a discrete design.

When the filtering technique is applied, the sensitivities of a function $F$ (objective or constraints) is evaluated w.r.t. the filtered densities $\bar{\rho}$. To obtain the sensitivities w.r.t. unfiltered densities $\rho$, the chain rule of differentiation is employed:

$$
\frac{\partial F}{\partial \rho_{e}}=\sum_{i \in N_{e}} \frac{\partial F}{\partial \bar{\rho}_{i}} \frac{\partial \bar{\rho}_{i}}{\partial \tilde{\rho}_{i}} \frac{\partial \tilde{\rho}_{i}}{\partial \rho_{e}}
$$

where $\partial \bar{\rho}_{i} / \partial \tilde{\rho}_{i}$ and $\partial \tilde{\rho}_{i} / \partial \rho_{e}$ can be obtained by differentiating (B.1) and (B.2).

\section{References}

[1] L. Meza, S. Das, J. Greer, Strong, lightweight, and recoverable three-dimensional ceramic nanolattices, Science 345 (2014) 1322-6.

[2] L. Meza, A. J. Zelhofer, N. Clarke, A. J. Mateos, D. Kochmann, J. Greer, Resilient 3D hierarchical architected metamaterials, Proc Nat Acad Sci USA 112 (37) (2015) 11502-11507.

[3] T. Schaedler, A. Jacobsen, A. Torrents, A. Sorensen, J. Lian, J. Greer, L. Valdevit, W. Carter, Ultralight Metallic Microlattices, Science 334 (6058) (2011) 962-965.

[4] S. Shan, S. Kang, J. Raney, P. Wang, L. Fang, F. Candido, J. Lewis, K. Bertoldi, Multistable Architected Materials for Trapping Elastic Strain Energy, Adv Mater 27 (2015) 4296-4301.

[5] K. Muamer, T. Frenzel, C. Findeisen, P. Gumbsch, M. Wegener, Tailored Buckling Microlattices as Reusable Light-Weight Shock Absorbers, Adv Mater 28 (2016) 5865-5870.

[6] O. Sigmund, J. S. Jensen, Systematic design of phononic band-gap materials and structures by topology optimization, Phil Trans R Soc Lond 361 (2003) 1001-1019. 
[7] D. Kochmann, K. Bertoldi, Exploiting Microstructural Instabilities in Solids and Structures: From Metamaterials to Structural Transitions, Appl Mech Rev 69 (2017) 050801-1.

[8] K. Bertoldi, P. M Reis, S. Willshaw, T. Mullin, Negative Poisson's Ratio Behavior Induced by an Elastic Instability, Adv Mater 22 (2010) 361-366.

[9] J. Paulose, A. S. Meeussen, V. Vitelli, Selective buckling via states of self-stress in topological metamaterials, Proc Nat Acad Sci USA $112(25)(2015) 7639-7644$.

[10] T. Schaedler, W. Carter, Architected Cellular Materials, Annual Review of Materials Research 46 (1) (2016) 187-210.

[11] J. Bauer, A. Schroer, R. Schwaiger, O. Kraft, Approaching theoretical strength in glassy carbon nanolattices, Nat Mater 15 (4) (2016) 438443.

[12] N. Triantafyllidis, M. W. Schraad, Onset of failure in aluminum honeycombs under general in-plane loading, J Mech Phys Solids 46 (6) (1998) 1089-1124.

[13] L. Meza, G. Phlipot, C. Portela, A. Maggi, L. C. Montemayor, A. Comella, D. Kochmann, J. Greer, Reexamining the mechanical property space of three-dimensional lattice architectures, Acta Mater 140 (2017) 424-432.

[14] F. G. Rammerstorfer, D. Pahr, T. Daxner, W. Vonach, Buckling in Thin Walled Micro and Meso Structures of Lightweight Materials and Material Compounds, Comput Mech 37 (2006) 470-478.

[15] R. Lakes, Materials With Structural Hierarchy, Nature 361 (6412) (1993) 511-515.

[16] X. Zheng, W. Smith, J. Jackson, B. Moran, H. Cui, D. Chen, J. Ye, N. Fang, N. Rodriguez, T. Weisgraber, C. Spadaccini, Multiscale metallic metamaterials, Nature Materials 15 (2016) 1100-1106.

[17] J. Guedes, N. Kikuchi, Preprocessing and postprocessing for materials based on the homogenization method with adaptive finite element methods, Comput Methods Appl Mech Engrg 83 (2) (1990) 143-198.

[18] N. Triantafyllidis, B. Maker, On the Comparison Between Microscopic and Macroscopic Instability Mechanisms in a Class of FiberReinforced Composites, J Appl Mech 52 (4) (1985) 794-800.

[19] G. Geymonat, S. Müller, N. Triantafyllidis, Homogenization of nonlinearly elastic materials, microscopic bifurcation and macroscopic loss of rank-one convexity, Arch Ration Mech Anal 122 (3) (1993) 231-290.

[20] N. Triantafyllidis, W. C. Schnaidt, Comparison of microscopic and macroscopic instabilities in a class of two-dimensional periodic composites, J Mech Phys Solids 41 (9) (1993) 1533-1565.

[21] B. Haghpanah, J. Papadopoulos, D. Mousanezhad, H. Nayeb-Hashemi, A. Vaziri, Buckling of regular, chiral and hierarchical honeycombs under a general macroscopic stress state, Proc Math Phys Eng Sci 470 (2167) (2014) 20130856.

[22] M. W. Schraad, N. Triantafyllidis, Scale Effects in Media With Periodic and Nearly Periodic Microstructures, Part I: Macroscopic Properties, J Appl Mech 64 (4) (1997) 763-771.

[23] J. Michel, O. Lopez-Pamies, P. Castaneda, N. Triantafyllidis, Microscopic and macroscopic instabilities in finitely strained porous elastomers, J Mech Phys Solids 55 (2007) 900-938.

[24] O. Sigmund, Materials with prescribed constitutuve parameters: An inverse homogenization problem, Int J Solids Struct 31 (17) (1994) 2313-2329.

[25] M. P. Bendsøe, O. Sigmund, Topology Optimization: Theory, Methods and Applications, Springer-Verlag, Berlin, 2003.

[26] O. Sigmund, S. Torquato, Design of Materials With Extreme Thermal Expansion Using a Three-Phase Topology Optimization Method, J Mech Phys Solids 45 (6) (1997) 1037-1067.

[27] U. D. Larsen, O. Sigmund, S. Bouwstra, Design and Fabrication of Compliant Micromechanisms and Structures with Negative Poisson's Ratio, J Microelectromech Syst 6 (2) (1997) 99-106.

[28] E. Andreassen, B. Lazarov, O. Sigmund, Design of manufacturable 3D extremal elastic microstructure, Mech Mater 69 (1) (2014) 1-10.

[29] A. Clausen, F. Wang, J. Jensen, O. Sigmund, J. Lewis, Topology Optimized Architectures with Programmable Poisson's Ratio over Large Deformations, Adv Mater 27 (37) (2015) 5523-5527.

[30] O. Sigmund, On the Optimality of Bone Microstructure, in: P. Pedersen, M. P. Bendsøe (Eds.), Synthesis in Bio Solid Mechanics, IUTAM, Kluwer, 221-234, 1999

[31] O. Sigmund, A new class of extremal composites, J Mech Phys Solids 48 (2) (2000) 397-428.

[32] M. P. Bendsøe, N. Triantafyllidis, Scale effects in the optimal design of a microstructured medium against buckling, Int J Solids Struct 26 (7) (1990) 725-741.

[33] M. M. Neves, O. Sigmund, M. Bendsøe, Topology optimization of periodic microstructures with a penalization of highly localized buckling modes, Int J Numer Meth Engng 54 (6) (2002) 809-834.

[34] M. M. Neves, O. Sigmund, M. Bendsøe, Topology optimization of periodic microstructures with a buckling criteria, Proceedings of the Fifth World Congress on Computational Mechanics, Vienna University of Technology, Austria, 2002.

[35] M. Osanov, J. Guest, Topology Optimization for Architected Materials Design, Annual Review of Materials Research 46 (1) (2016) $211-233$.

[36] M. M. Neves, H. Rodrigues, J. M. Guedes, Generalized topology design of structures with a buckling load criterion, Struct Optim 10 (2) (1995) 71-78.

[37] A. P. Seyranian, E. Lund, N. Olhoff, Multiple eigenvalues in structural optimization problems, Struct Optim 8 (1994) 207-227.

[38] R. M. Gorguluarslan, U. N. Gandhi, R. Mandapati, S.-K. Choi, Design and fabrication of periodic lattice-based cellular structures, Comput Aided Des Appl 13 (1) (2016) 50-62.

[39] A. Tyas, M. Gilbert, T. Pritchard, Practical plastic layout optimization of trusses incorporating stability considerations, Comput Struct 84 (2006) $115-126$.

[40] P. Dunning, E. Ovtchinnikov, J. Scott, H. A. Kim, Level-set topology optimization with many linear buckling constraints using an efficient and robust eigensolver, Int J Numer Meth Engng 107 (2016) 1029-1053.

[41] R. Kemmler, A. Lipka, E. Ramm, Large deformations and stability in topology optimization, Struct Multidiscipl Optim 30 (2005) 459-476.

[42] E. Lindgaard, E. Lund, A unified approach to nonlinear buckling optimization of composite structures, Comput Struct 89 (2011) 357-370.

[43] M. Jalalpour, T. Igusa, J. Guest, Optimal design of trusses with geometric imperfections: Accounting for global instability, Int J Solids Struct 48 (2011) 3011-3019. 
[44] M. Jansen, G. Lombaert, M. Schevenels, Robust topology optimization of structures with imperfect geometry based on geometric nonlinear analysis, Comput Methods Appl Mech Engrg 285 (2015) 452-467.

[45] L. Brillouin, Wave Propagation in Periodic Structures: Electric Filters and Crystal Lattices, Dover, New York, 1953.

[46] O. Sigmund, K. G. Hougaard, Geometric properties of optimal photonic crystals, Phys Rev Lett 100 (15) (2008) 153904, ISSN 10797114, 00319007.

[47] C. Thomsen, Buckling Optimization of Extremal Materials (MSc thesis), Department of Mechanical Engineering, Technical University of Denmark, 2017.

[48] K. Svanberg, The method of moving asymptotes - A new method for structural optimization, Int J Numer Meth Engng 24 (1987) 359-373.

[49] F. Wang, J. Jensen, O. Sigmund, Robust topology optimization of photonic crystal waveguides with tailored dispersion properties, J Opt Soc Am B 28 (3) (2011) 387-397.

[50] J. Guest, Topology optimization with multiple phase projection, Comput Methods Appl Mech Engrg 199 (1-4) (2009) 123-135. 